\title{
Nouvelles données sur l'assemblage lithique de la couche L2/3 de La Micoque (Les Eyzies-de- Tayac, Dordogne, France)
}

New data about lithic components of the layer L2/3 from La Micoque (Les Eyziesde-Tayac, Dordogne, France)

Cyrielle Mathias, Sophie Grégoire et Marie-Hélène Moncel

\section{OpenEdition \\ Journals}

Édition électronique

URL : http://journals.openedition.org/paleo/3408

DOI : $10.4000 /$ paleo.3408

ISSN : $2101-0420$

Éditeur

SAMRA

Édition imprimée

Date de publication : 30 décembre 2017

ISSN : 1145-3370

\section{Référence électronique}

Cyrielle Mathias, Sophie Grégoire et Marie-Hélène Moncel, « Nouvelles données sur l'assemblage lithique de la couche L2/3 de La Micoque (Les Eyzies-de-Tayac, Dordogne, France) », PALEO [En ligne], 28 | 2017, mis en ligne le 01 juin 2018, consulté le 07 juillet 2020. URL : http://

journals.openedition.org/paleo/3408; DOI : https://doi.org/10.4000/paleo.3408

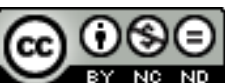

PALEO est mis à disposition selon les termes de la licence Creative Commons Attribution - Pas d'Utilisation Commerciale - Pas de Modification 4.0 International. 


\title{
Nouvelles données sur l'assemblage lithique de la couche L2/3 de La Micoque (Les Eyzies-de-Tayac, Dordogne, France)
}

\author{
Cyrielle MATHIAS (a), Sophie GRÉGOIRE(a) \\ et Marie-Hélène MONCEL ${ }^{(a)}$
}

Résumé : Le gisement de La Micoque (Les Eyzies-de-Tayac, Dordogne), s’il est surtout connu comme éponyme des techno-complexes micoquiens et tayaciens, a également livré des niveaux attribuables aux premières phases du Paléolithique moyen en Périgord. Au sein de cette riche séquence, la couche L2/3, corrélée au MIS 10 et caractérisée par F. Bordes comme de l'Acheuléen méridional, livre une industrie orientée quasi exclusivement vers la production d'éclats.

D'après l'étude technologique, plusieurs chaînes opératoires de production d'éclats ont ainsi pu être identifiées, simples ou ramifiées. Une production originale de supports allongés à dos et à tranchants linéaires coexiste avec des productions diversifiées issues de débitages sur éclat et de débitages Levallois. Le façonnage est très peu utilisé avec une seule pièce bifaciale. Les produits retouchés montrent une recherche de tranchants linéaires à l'instar de la production brute dominante, tandis que les contacts punctiformes sont rares.

L'assemblage lithique de la couche L2/3 nous permet ainsi de renseigner une autre facette de la variabilité inhérente à ces sites du Paléolithique moyen ancien.

Mots-clés : Technologie lithique, Paléolithique moyen ancien, Micoque, Sud-Ouest, France.

Abstract: New data about lithic components of the layer L2/3 from La Micoque (Les Eyzies-de-Tayac, Dordogne, France). The site of La Micoque (Les Eyzies-de-Tayac, Dordogne), is known as the Micoquian and the Tayacien techno-complexes's type site. The site also yielded levels from the early Middle Palaeolithic in the Perigord. In this rich sequence, the layer L2/3, correlated to MIS 10 and described by F. Bordes as a Southern Acheulean, yielded an industry almost exclusively turned towards the production of flakes.

Following the technological analysis, several reduction strategies of productions of flakes have been identified among the core technology processes, unique of ramified. An original production of elongated backed blanks and products with linear cutting edges coexists with diversified core technologies such as cores on flakes or Levallois cores. The shaping is rare with only one bifacial tool. A search of linear edges is highlighted by the study of retouched flakes, as in the main production. The point-like edges are rare.

The lithic assemblage of the layer L2/3 allows us describing another aspect of the inherent variability the early Middle Paleolithic in Western Europe.

Key-words: Lithic technology, Early Middle Palaeolithic, Micoque, South-West Europe.

(a) UMR 7194 - Histoire Naturelle de l'Homme Préhistorique - UPVD-MNHN-CERPT - cyrielle.mathias@gmail.com ; sophie.gregoire@cerptautavel.com; moncel@mnhn.fr 


\section{Introduction}

En Europe, les premières manifestations du Paléolithique moyen sont souvent liées à l'émergence de nouvelles méthodes de débitage, en particulier celles associées au concept Levallois, ainsi qu'à la disparition des bifaces (Bordes 1950). Les données actuelles indiquent que le Paléolithique moyen ancien (MIS 10 à 6) est bien plus diversifié et ne peut se réduire à cette seule apparition du débitage Levallois et à la disparition progressive des bifaces. Ainsi, durant cette phase initiale du Paléolithique moyen ancien, de nombreux systèmes de production d'éclats et de façonnage coexistent au sein des séries lithiques. La variabilité des assemblages de cette période est attestée tant sur le plan régional que chronologique dans le Sud de la France (Brenet et al. 2008 ; Colonge et al. 2010 ; Brenet et al. 2014 ; Moncel et al. 2014 ; Hérisson et al. 2016 ; Carmignani et al. 2017). Les éléments pouvant expliquer la diversité dans la composition des assemblages lithiques sont aussi bien d'ordre économique que fonctionnel (contexte pétrographique, fonction du site, durée d'occupation) ou environnemental (contexte climatique, périodes glaciaires ou interglaciaires) ou encore culturel (traditions régionales, invention ou diffusion de phénomènes techniques). La durée de la période au sein de laquelle se développent les occupations du Paléolithique moyen ancien - soit entre les MIS 10 et 6 - ainsi que l'aire géographique concernée - européenne - rendent complexe l'appréhension des facteurs de cette variabilité.

En Dordogne, peu d'indices d'occupations sont recensés pour le Pléistocène moyen avant le MIS 10. Pour la période du Paléolithique moyen ancien juste postérieure, le nombre est plus important. Ces occupations sont localisées principalement dans trois zones : la vallée de l'Isle au nord, la vallée de la Vézère et la vallée de la Dordogne au sud (Sarladais et Bergeracois).

Des occupations du MIS 9 et 8 sont ainsi présentes à PetitBost (Bourguignon et al. 2008). A proximité, le site des Tares a été rattaché sur des bases géoarchéologiques aux MIS $9 / 8$, bien que non daté par des méthodes radiométriques (Rigaud et Texier 1981 ; Geneste et Plisson 1996). Les niveaux inférieurs de la grotte Vaufrey, même s'ils ne renferment que peu de matériel lithique, pourraient être attribués au MIS 8 voire au MIS 10. Par ailleurs, un Moustérien décrit comme typique est présent dans les niveaux des MIS 7 à 5 du gisement (Rigaud 1988 ; Hernandez et al. 2014).

À partir du MIS 7 et au MIS 6, les niveaux d'occupations sont de plus en plus nombreux. On peut mentionner par exemple les sites du Pech de l'Azé II c. 6 à 9 (Bordes 1971), les niveaux inférieurs de Combe-Grenal (Bordes 1971), Combe-Brune 2 (Brenet et al. 2008 ; Brenet 2011 ; Frouin et al. 2014), Cantalouette 1, Combe-Brune 3 (Brenet et al. 2006) ou encore Barbas C'4, C'3 base (Boëda et Kerzavo 1991 ; Boëda et al. 1996 ; Valladas et al. 1999). Parmi ces sites coexistent des industries attribuées tantôt à l'Acheuléen, à l'Acheuléen méridional (Bordes 1971) ou au Paléolithique moyen ancien.

Le site de La Micoque s'intègre dans les phases anciennes du Paléolithique moyen et de manière générale parmi les plus anciens gisements stratifiés du Périgord par la présence de niveaux plus anciens que le MIS 7.

\section{1 - Le gisement de La Micoque}

\section{1 - Cadre géographique et historique des recherches}

Le gisement de La Micoque ${ }^{1}$ est situé sur la commune des Eyzies-de-Tayac, en Dordogne (Nouvelle-Aquitaine ; fig. 1). Il est situé sur le flanc gauche de la vallée du Manaurie (affluent de la Vézère), à une vingtaine de mètres audessus du lit du ruisseau. La vallée est creusée dans les calcaires du Coniacien, tandis que le plateau est recouvert d'altérites, reprises par colluvionnement sur les versants (Texier 2006).

À l'origine, rien ne laissait présager un gisement paléolithique en ce lieu puisqu'il se présentait comme un talus en pente de $30^{\circ}$. On doit les premiers signalements à l'exploitant agricole qui montra du matériel recueilli en surface à $P$. Fournier, alors en train de fouiller LaugerieHaute à $500 \mathrm{~m}$ de là (Peyrony 1938). Ce dernier fit alors part de l'information à $E$. Rivière. Les premières fouilles du gisement remontent à l'année 1896 et ont été effectuées par G. Chauvet et E. Rivière (Chauvet et Rivière 1896) d'une part puis par L. Capitan (Capitan 1896). À la fin du $\mathrm{XIX}$, des fouilles ont également été réalisées par $\mathrm{E}$. Harlé (1897) et $D$. Peyrony (1898). Au début du $X X^{\ominus}$ siècle, les fouilleurs sont toujours aussi nombreux : L. Coutil de 1903 à 1905, E. Cartailhac en 1905, O. Hauser en 1906 et 1907, des scientifiques berlinois en 1912 (Rosendahl 2006) et enfin de nouveau D. Peyrony de 1929 à 1932. Dans les années 50, F. Bordes entreprend également des recherches sur le gisement par le biais d'un sondage (1956; Bordes 1984).

Après une reprise générale de la stratigraphie par $\mathrm{H}$. Laville et J.-Ph. Rigaud (Laville 1975 ; fig. 2), plusieurs campagnes de fouilles ont été menées par J.-Ph. Rigaud et A. Debénath de 1983 à 1997 (Debénath et Rigaud 1988 ; Debénath et al. 1991). Le matériel étudié dans ce travail est issu de ces dernières campagnes de terrain, réalisées selon une méthodologie rigoureuse.

L'histoire du site et de son exploitation rendent par conséquent complexes les tentatives de correspondances entre certaines collections. Un travail de corrélations stratigraphiques, notamment entre les données de $D$. Peyrony et d'O. Hauser a déjà été réalisé (Rosendahl 2006).

(1) Du nom de l'ancienne ferme érigée à $500 \mathrm{~m}$ du site (Peyrony 1938). 


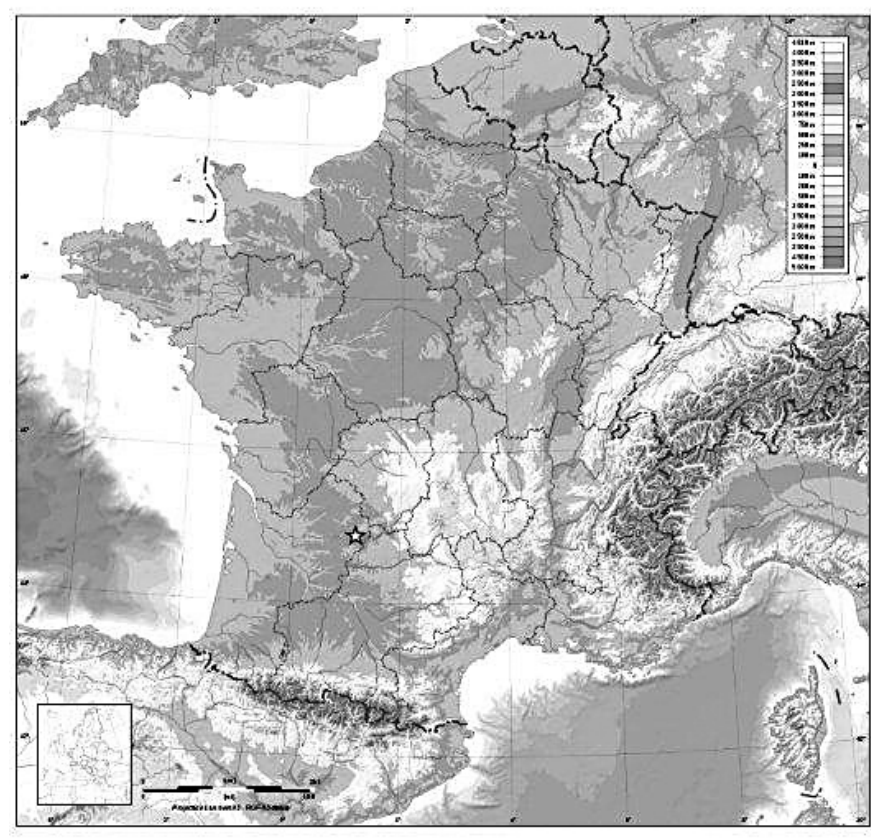

Figure 1 - Localisation du site de La Micoque.

Figure 1 - Location of the site of La Micoque.

En ce qui concerne le matériel qui nous intéresse ici, le niveau L2/3 des fouilles d'A. Debénath et J.-Ph. Rigaud, il correspondrait à la couche 5 et à la subdivision $L$ de la seconde stratigraphie de D. Peyrony (Peyrony 1938) ainsi qu'à la couche 5' de F. Bordes. II semble également correspondre aux subdivisions $\mathrm{F}$ et $\mathrm{G}$ d'O. Hauser (Hauser 1916 ; Rosendahl 2006).

\section{2 - Mise en place des dépôts et contexte chronologique}

Trois types de processus sédimentaires sont à l'origine de la mise en place des dépôts de La Micoque : des coulées de débris, des phénomènes gravitaires (éboulis) et des processus fluviatiles (largement dominants) (Texier et Bertran 1993 ; Texier 2006). D'après ces auteurs, la séquence comporte trois ensembles sédimentaires. Les ensembles inférieur et moyen (couches $\mathrm{A}$ à $\mathrm{N}$ de Peyrony) correspondent à des terrasses emboîtées du Manaurie édifiées lors de périodes froides et arides tandis que l'ensemble supérieur (non-identifié antérieurement) correspond à des colluvions holocènes.

À partir de l'analyse de la dynamique de dépôt, ils proposent un certain nombre d'hypothèses paléoenvironnementales. Ainsi, les dépôts alluviaux témoignent du fonctionnement d'une rivière à chenaux tressés avec un très fort débit concentré sur une courte période de l'année et présentant un écoulement faible à très faible pendant le reste de l'année. Les structures lamellaires identifiées dans les rares dépôts limoneux de l'ensemble moyen sont interprétées comme le résultat de la

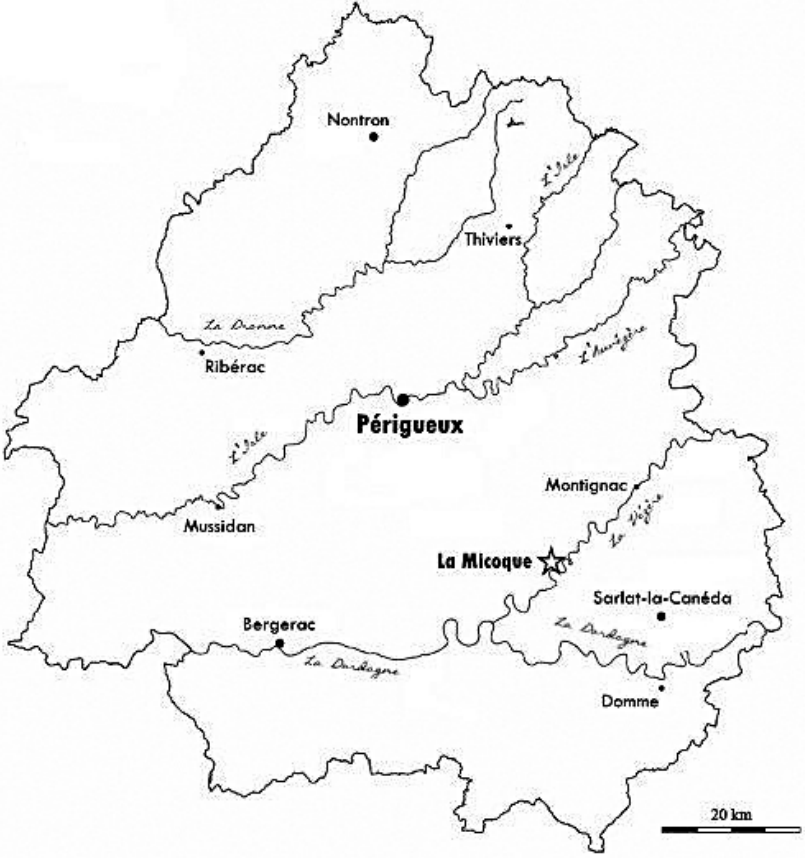

formation de lentilles de glace de ségrégation et témoignent donc d'un contexte climatique froid (Texier 2006).

Du point de vue chronologique, les nappes alluviales des ensembles inférieur et moyen ont été corrélées respectivement avec les nappes Fw1 et Fw2 de la Vézère (Texier 2006). D'après les données recueillies dans les différentes vallées du nord de l'Aquitaine, cet auteur attribue la nappe la plus ancienne au MIS 12, entre 470 et $440 \mathrm{Ka}$, et la nappe la plus récente au MIS 10, entre 370 et $350 \mathrm{Ka}$. Le surcreusement qui les sépare de même que les alluvions argileuses situées à la base de l'ensemble moyen sont rapportés au MIS 11 (440-370 Ka).

Ces hypothèses chronologiques sont en partie corroborées par les datations radiométriques les plus récentes réalisées pour l'ensemble moyen (Falguères, Bahain et Saleki 1997). Les niveaux $E$ à $L$ peuvent être attribués à une période comprise entre 350 et $300 \mathrm{ka}$, au MIS 10 lors d'une période semi-aride froide (Falguères, Bahain et Saleki 1997 ; Texier et Bertran 1993). Falguères, Bahain et Saleki (1997) ont ainsi obtenu trois dates par ESR/U-Th pour la couche L2/3 : $313 \pm 47,293 \pm 44$ et $291 \pm 44 \mathrm{Ka}$. Les auteurs concluent que ces dates confirment l'hypothèse émise par Texier et Bertran (1993) d'un dépôt au cours du MIS 10.

\section{3 - Occupations humaines à La Micoque}

La séquence de La Micoque est connue comme éponyme de deux techno-complexes du Pléistocène moyen d'Europe de l'Ouest : le Micoquien (couche 6) et le Tayacien (couches 4 et 5 ) définis par l'Abbé $H$. Breuil d'après le matériel des fouilles Peyrony (Breuil 1932). 

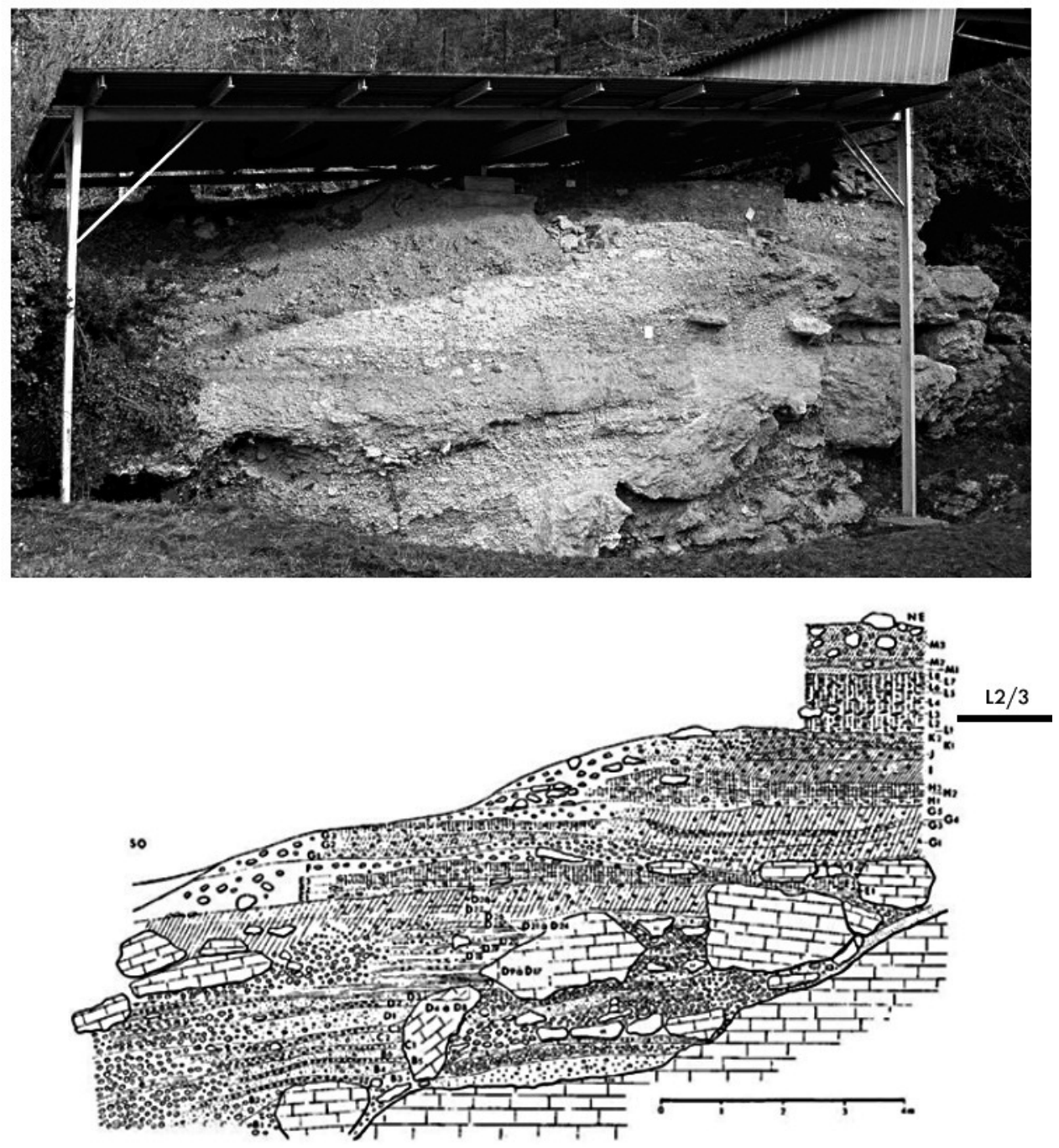

Figure 2 - Photographie de la stratigraphie de La Micoque, relevé Laville et Rigaud (in : Laville1975) et position de la couche L2/3.

Figure 2 - Photograph of the stratigraphical sequence, drawing from Laville et Rigaud (in: Laville 1975) and location of the layer L2/3.

Toutefois, les altérations taphonomiques (et particulièrement mécaniques) affectant certaines couches altèrent leur identification, notamment pour la présence d'encoches et de denticulés caractérisant le Tayacien.

Lors des premières fouilles, G. Chauvet et E. Rivière qualifient l'industrie mise au jour de " chélléomoustérienne ", en raison de la découverte de plusieurs types de " haches " associées à des racloirs moustériens (Chauvet et Rivière 1896). Ce sont ces pièces, des bifaces amygdaloïdes, qui caractériseront le Micoquien de la couche 6.

Dans les années 1930, H. Breuil et D. Peyrony redéfinissent les types d'industries (Breuil 1932 ; Peyrony 1938). F. Bordes reprend également l'étude du site et donne ses propres attributions culturelles des séries (Bordes 1969) (tabl. 1). Les derniers travaux sur La Micoque ont permis de retrouver la plupart des couches archéologiques décrites antérieurement à l'exception de la couche Micoquienne qui se trouverait en position terminale à la base des colluvions holocènes.
La production lithique de la couche L2/3 a été déterminée comme à débitage d'éclats et rares bifaces (Delpech et al. 1995).

\section{4 - Matériel et méthode}

Le matériel lithique étudié est issu de la couche $L 2 / 3$ des fouilles A. Debénath/J.-Ph. Rigaud dont le matériel est conservé au Musée National de Préhistoire des Eyzies-deTayac. L'étude a été réalisée sur l'ensemble du matériel, incluant le matériel coordonné et celui issu des refus de tamis.

Une analyse technologique visant à déterminer les objectifs de production a été réalisée. Elle a pour but de mettre en évidence les concepts de débitage et de façonnage mis en œuvre pour répondre à la production des supports recherchés. Ces concepts peuvent être variés au Paléolithique moyen ancien. Concernant les modes de production d'éclats, on retrouve principalement des débitages S.S.D.A. (Ashton 1992 ; Forestier 1992, 1993), 


\begin{tabular}{cccc} 
Couche & $\begin{array}{c}\text { H. Brevil } \\
(1932)^{*}\end{array}$ & $\begin{array}{c}\text { D. Peyrony } \\
(1938)^{*}\end{array}$ & $\begin{array}{c}\text { F. Bordes } \\
(1969)^{*}\end{array}$ \\
\hline $\mathbf{6}(\mathbf{N})$ & Micoquien & Micoquien & Micoquien \\
\hline $\mathbf{5} ; \mathbf{5}^{\prime}(\mathrm{J})$ & Tayacien & Tayacien & Acheuléen méridional \\
\hline $\mathbf{4}(\mathbf{H})$ & Tayacien & Vieux Moustérien classique & Prémoustérien \\
\hline $\mathbf{3 ( E )}$ & Clactonien & Tayacien & Prémoustérien \\
\hline $\mathbf{2 ( C )}$ & Clactonien & Tayacien, débitage clactonien & \\
\hline $\mathbf{1}(\mathbf{A})$ & Clactonien & Tayacien, débitage clactonien & Clactonien \\
\hline
\end{tabular}

Tableau 1 - Attribution culturelles des niveaux de la Micoque. * : déterminations réalisées d'après leurs propres collections.

Table 1 - Cultural attribution of the layers of la Micoque. * : determinations on their own collections.

Levallois (Boëda 1986), Discoïde (Boëda 1993 ; Peresani 1998) ou encore Quina (Bourguignon 1997) et Trifacial (Boëda 1997 ; Chevrier 2006). Ces production peuvent être simples, complémentaires ou ramifiées (Bourguignon, Faivre et Turq 2004 ; Faivre 2008).

Les pièces confectionnées (retouchées et façonnées) ont été analysées selon une approche techno-morphofonctionnelle développée depuis les années 1990 (Lepot 1993 ; Bourguignon 1997 ; Boëda 1997, 2013). Ce type d'analyse vise à mettre en exergue les parties constitutives de l'outil (transformatives, réceptrices de l'énergie et préhensives) et la façon dont elles sont agencées sur le support (structure). En s'intéressant au tranchant et non pas à la seule localisation de la retouche sur le support, on appréhende plus précisément les parties recherchées sur l'outil (Boëda 2013). D'après les récurrences et les ruptures sur l'outil sont définies ainsi différentes unités : des Unité Techno-Fonctionnelles ${ }^{2}$ de Contact Transformatif (UTF CT) et de Contact Préhensif/Réceptif de l'énergie (UTF CP/CR). Dans certains cas, ces unités peuvent être considérées comme bivalentes (Viallet 2016), c'est-à-dire que la zone de contact, de par sa morphologie ainsi que son angulation, peut correspondre à une zone de maintien ou à une zone transformative. Ces zones sont nécessairement associées à une autre zone de contact transformatif.

\section{2 - Résultats. Analyse technologique de la couche L2/3}

\section{1 - Matières premières employées}

Si l'assemblage est largement dominé par l'utilisation du silex (98,83\%, $n=3869$, tabl. 2), la désilicification avancée rend complexe voire impossible la détermination précise du type employé. D'après l'observation des cortex, des silex alluviaux et issus des altérites ont été utilisés, très probablement des affleurements du Sénonien au sens large de la vallée de la Vézère (fig. 3 - 1 et 2).

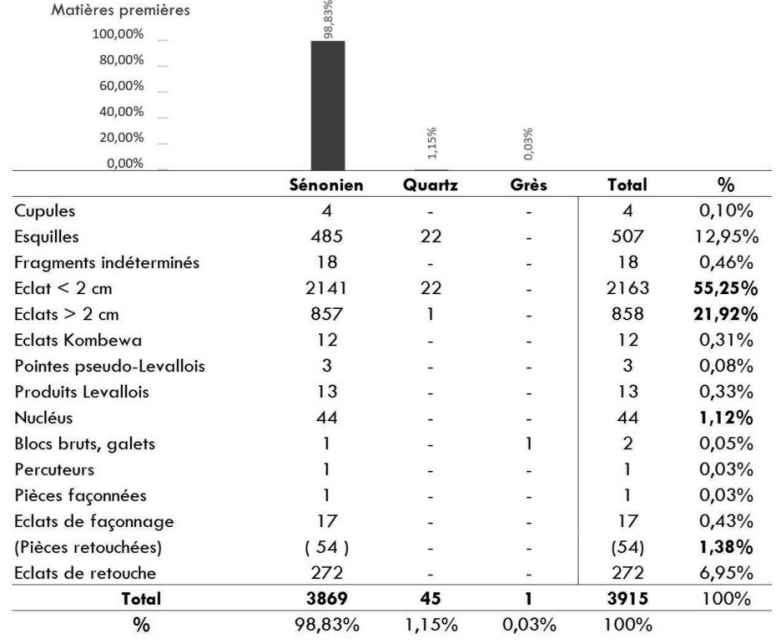

Tableau 2 - Décompte du matériel lithique de la couche L2/3 selon les matières premières.

Table 2 - Count of the lithic material from layer 2/3 according to raw materials.

Malgré une patine systématique et totalement couvrante, des macro-fossiles sont visibles à l'œil nu sur un grand nombre de pièces. II s'agit essentiellement de Bryozoaires et d'ostracodes, généralement pris dans de la silice micritique de texture mudstone (fig. 3 - B) parfois packstone à nombreux pellets et intraclastes roulés (fig. 3 - C). Ces fossiles semblent suggérer l'utilisation de faciès du Crétacé supérieur (Séronie-Vivien et Séronie-Vivien 1987 ; fig. 3 : A, B et C).

Deux types de quartz ont également été utilisés même s’ils restent largement minoritaires (fig. $3-3$ à 5). Le premier correspond à un quartz dont les cristaux sont grossiers mais semblant disposer de bonnes qualités à la taille. II est marqué par des imprégnations d'oxydes de fer. Le second est un quartz blanc translucide à cristaux plus fins.

Enfin, un galet fracturé en grès grossier est présent dans l'assemblage. L'altération des surfaces ne permet pas d'identifier d'éventuels stigmates.

\section{2 - Données générales sur l'assemblage}

L'étude a porté sur l'ensemble du matériel issu des fouilles d'A. Debénath et J.-Ph. Rigaud, soit un total de 3915 pièces, essentiellement composées d'éclats et d'esquilles (tabl. 2). L'ensemble du matériel a été récolté (coordonné et refus de tamis), et on retrouve ainsi dans l'assemblage de nombreux éclats de retouche, des esquilles et des débris.

Bien que le matériel soit fortement désilicifié, il n'a que rarement subi une altération gênant la lecture, à l'inverse

(2) E. Boëda définit une Unité Techno-Fonctionnelle comme " un ensemble d'éléments et/ou caractères techniques qui coexistent dans une synergie d'effets " (Boëda 1997 - p. 34). 


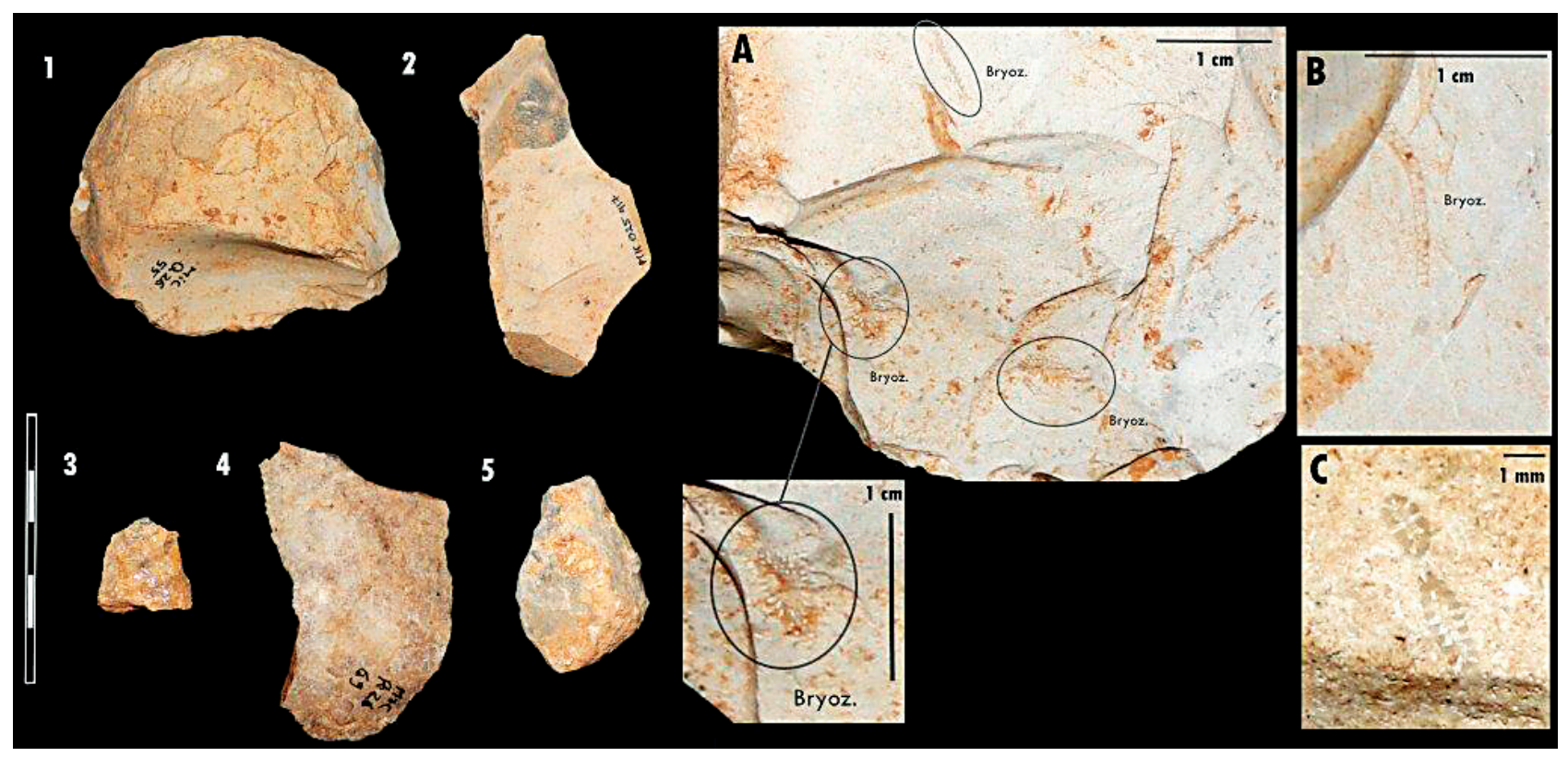

Figure 3 - Photographies des matières premières utilisées. 1 et $2:$ silex du Sénonien, 3,4 et $5:$ deux types de quartz. A, B et $C$ : bioclastes du silex du Crétacé supérieur. Bryoz : Bryozoaire.

Figure 3 - Photographs of raw materials used. 1 and 2 : Senonian Flint, 4,5 and $6:$ two types of quartz. A, B and $C:$ bioclasts from the upper Cretaceous flint. Bryoz . : bryozoans.

des niveaux sus-jacents et sous-jacents dont les pièces présentent un aspect fortement roulé.

La présence des éléments les plus petits (esquilles, éclats de retouche, fragments d'éclats) indique une mise en place des dépôts ayant faiblement remanié le matériel. Deux remontages ont par ailleurs été effectués, sans qu'une recherche systématique ait été réalisée.

Différentes étapes des chaînes opératoires semblent présentes sur le site puisque 18,9\% des éclats présentent des résidus de cortex (présence d'éclats à cortex résiduel, d'éclats semi-corticaux et d'éclat corticaux dont des éclats d'entame entièrement en cortex ; tabl. 3). Les différents débitages sont réalisés indifféremment sur des blocs et des galets de silex (pas de sélection préférentielle). Les rares pièces en quartz montrent potentiellement une chaîne opératoire de débitage segmentée (dans le temps et l'espace ?). Si l'hypothèse de percuteurs fracturés est envisageable (esquilles résultant de la fracturation), de véritables éclats sont toutefois présents dans la série. Très peu d'éclats portent des restes de cortex (13,3\%) alors qu'il est reconnu que les zones corticales offrent de meilleures surfaces de plan de frappe pour ce matériau (Tavoso 1978). Le caractère incomplet de la chaîne de débitage sur quartz est induit par l'absence de nucléus dans la série.

\section{3 - Systèmes de débitage identifiés}

Le débitage du niveau L2/3 se caractérise par la recherche de produits allongés à dos. Ainsi, 70,6\% des éclats entiers ont un indice d'allongement supérieur à 1 et $33,6 \%$ supérieur à 1,5 (fig. 4). Nous distinguons ces éclats

\begin{tabular}{lccccc}
\hline & Silex & $\%$ & Quartz & $\%$ & Total \\
\hline Sans cortex & 3085 & $81,1 \%$ & 39 & $86,7 \%$ & 3124 \\
A cortex résiduel & 340 & $8,9 \%$ & 2 & $4,4 \%$ & 342 \\
Semi-cortical & 183 & $4,8 \%$ & 2 & $4,4 \%$ & 185 \\
Cortical & 198 & $5,2 \%$ & 2 & $4,4 \%$ & 200 \\
\hline Total & 3806 & $100,0 \%$ & 45 & $100 \%$ & $\mathbf{3 8 5 1}$ \\
\hline
\end{tabular}

Tableau 3 - Caractéristiques des faces supérieures d'éclats.

Table 3 - Characteristics of the dorsal surface of flakes.

allongés des lames dont l'indice d'allongement est supérieur à $2(L=2 x l)$. Outre leur rapport $L / l$, les lames attestent également de l'utilisation de concepts de débitage spécifiques.

Parallèlement à cette recherche dominante, plusieurs concepts de débitage cohabitent et montrent une variété plus grande dans les produits recherchés. Les débitages algorithmiques au sens large (Ashton 1992 ; Forestier 1993) sont les plus représentés par les nucléus (33,3\% ; $n=14)$, suivis par les concepts de débitage sur éclats $(23,8 \% ; n=10)$ et le débitage Levallois $(16,7 \% ; n=7)$. Quelques blocs testés $(n=6)$ ainsi que des nucléus indiquant des débitages alternants $(n=3)$ complètent la série (tabl. 4).

\section{Les débitages algorithmiques et unipolaires allongés}

Les productions algorithmiques (clactoniennes ou S.S.D.A., Ahston 1992 ; Forestier 1992, 1993 ; tabl. 5 ; pl. 2) peuvent être divisées en plusieurs catégories, en fonction du type de 


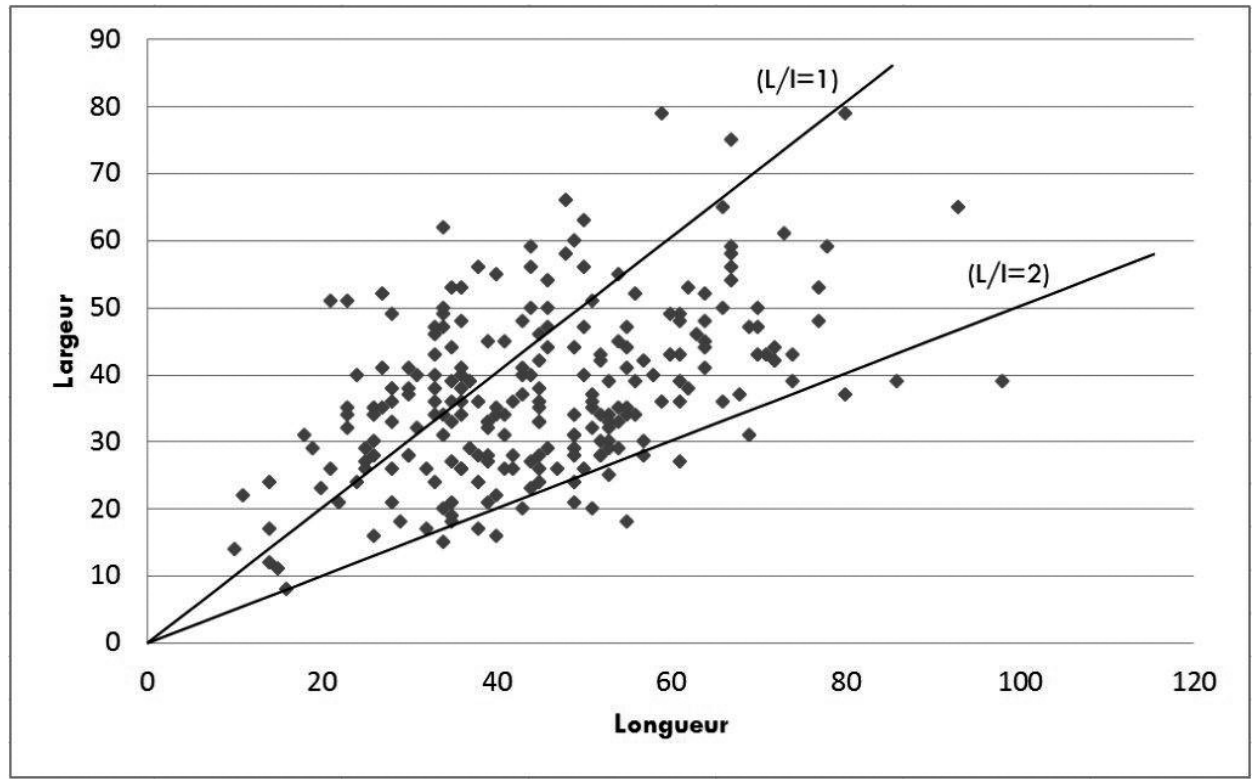

Figure 4 - Allongement des éclats entiers.

Figure 4 - Elongation of entire flakes.

\begin{tabular}{lcc} 
Débitages identifiés d'après les nucléus & Silex & $\%$ \\
\hline Débitages sur éclat & 10 & $22,7 \%$ \\
Hiérarchisé non-Levallois & 4 & $9,1 \%$ \\
Levallois & 7 & $15,9 \%$ \\
S.S.D.A. ; unipolaire allongé & 14 & $31,8 \%$ \\
Débitage alternant (non discoïde) & 3 & $6,8 \%$ \\
Bloc testé, ébauches & 6 & $13,6 \%$ \\
\hline Total & $\mathbf{4 4}$ & $100 \%$ \\
\hline
\end{tabular}

Tableau 4 - Débitages identifiés d'après les nucléus.

Table 4 - Flaking methods identified following the cores.

produit recherché mais aussi en fonction du nombre de surfaces de débitage et/ou de plan de frappe ainsi que du volume utile exploité par rapport au volume global du nucléus.

Les produits principalement recherchés sont des éclats laminaires à dos majoritairement corticaux (ils peuvent occasionnellement être semi-corticaux ou bruts de débitage). Ces éclats sont en majorité obtenus dans le cadre d'un débitage algorithmique unipolaire ou bipolaire (pl. 1). Les convexités latérales ne semblent pas aménagées d'après les négatifs visibles sur les faces supérieures des éclats et seul le plan de frappe est aménagé. La présence d'un dos cortical étant recherchée, le débitage est latéralisé. Si cette production est très bien représentée parmi les éclats, il existe une sousreprésentation des nucléus associés à cette production. En effet, seules quelques séquences unipolaires allongées sont attestées par des nucléus à surfaces hiérarchisées non-Levallois (plan de frappe aménagé, débitage sécant à subparallèle, absence d'aménagement des convexités) ou des séquences brèves unipolaires sur quelques nucléus. Les séquences unipolaires ou unipolaires avec la recherche de produits allongés ont lieu généralement sur

\begin{tabular}{lcc}
\hline Méthodes algorithmiques et unipolaires allongées & Silex & $\%$ \\
\hline Unipolaire allongée à 2 surfaces de débitage & 2 & $12,5 \%$ \\
Unipolaire à 1 ou 2 surfaces de débitage & 3 & $18,8 \%$ \\
Unipolaire hiérarchisée & 2 & $12,5 \%$ \\
Bipolaire à 2 surfaces de débitage & 2 & $12,5 \%$ \\
Autres séquences à 2 surfaces de débitage ou plus & 5 & $31,3 \%$ \\
Méthodes variées à 4 surfaces ou plus & 2 & $12,5 \%$ \\
\hline Total & 16 & $100 \%$ \\
\hline
\end{tabular}

Tableau 5 - Méthodes algorithmiques et unipolaires allongées d'après les nucléus.

Table 5 - Algorithmic methods and elongated unipolar flaking following the cores.

deux surfaces (dont une sert ensuite de plan de frappe). Des productions dont les objectifs sont similaires sont réalisées successivement sur le nucléus (e.g. gestion matricielle successive identique). Dans quelques cas $(n=2)$, les surfaces sont hiérarchisées et le débitage, sécant, a lieu préférentiellement sur une surface, témoignant d'un investissement plus important par rapport au volume initial du nucléus.

Deux remontages, comportant à chaque fois deux éclats, attestent de ce type de débitage (pl. 1, A : 025-523 et 526 ; B : P25-61a et b).

Seuls deux nucléus témoignent d'une exploitation bipolaire à deux surfaces de débitage, la majorité des nucléus algorithmiques renvoie à des exploitations mixtes correspondant à des gestions matricielles successives différenciées $(n=5)$. Là encore, ce sont majoritairement deux surfaces qui sont exploitées suivant des associations diverses entre les débitages unipolaires, bipolaires, centripètes ou encore croisés. Ce type d'exploitation ne confère pas aux nucléus de morphologies spécifiques. Les produits recherchés sont variés (à dos, quadrangulaires, plus larges que longs). 
A
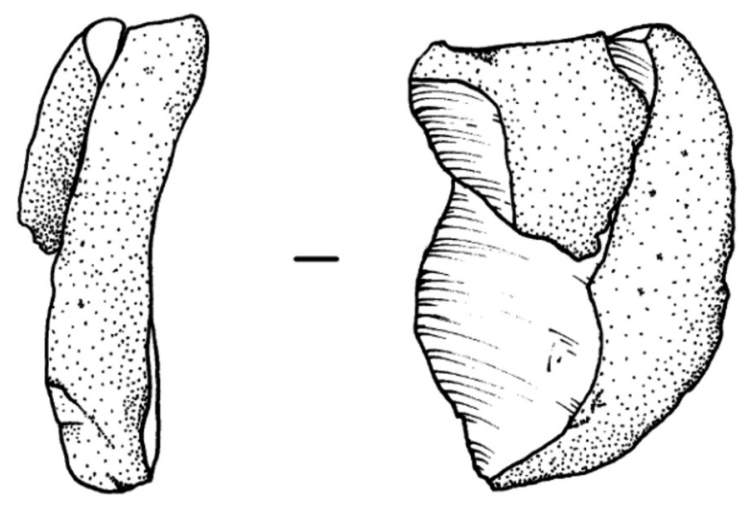

B

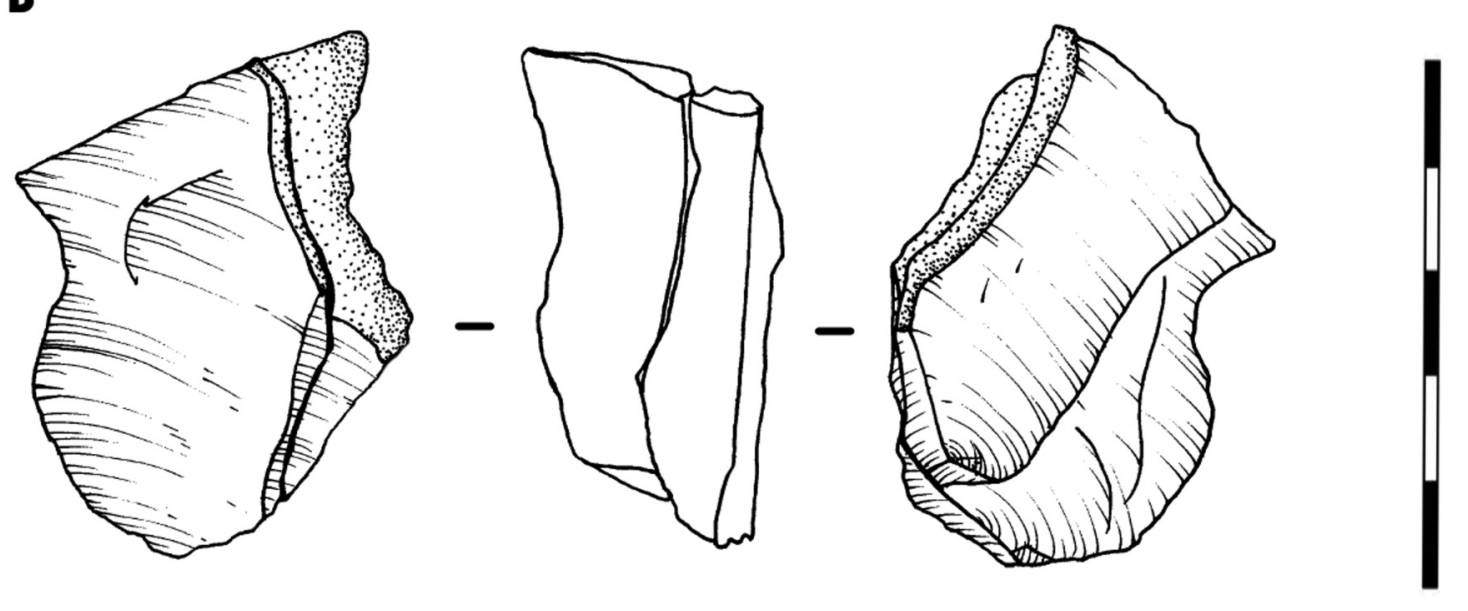

C

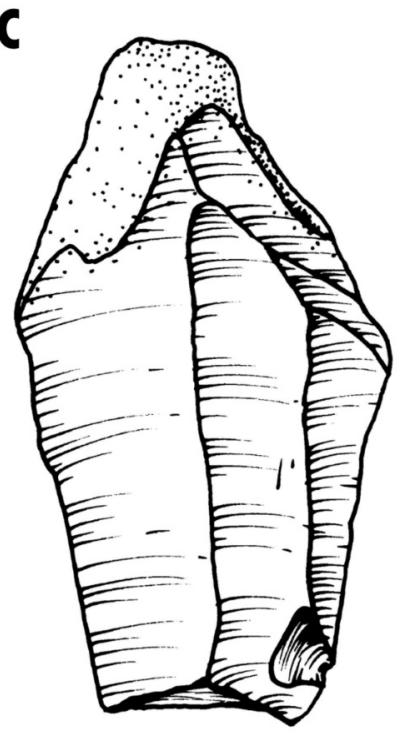

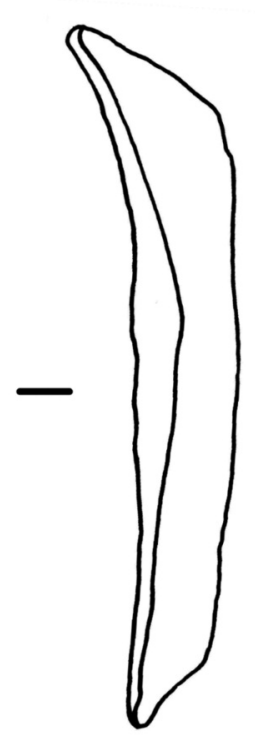

D

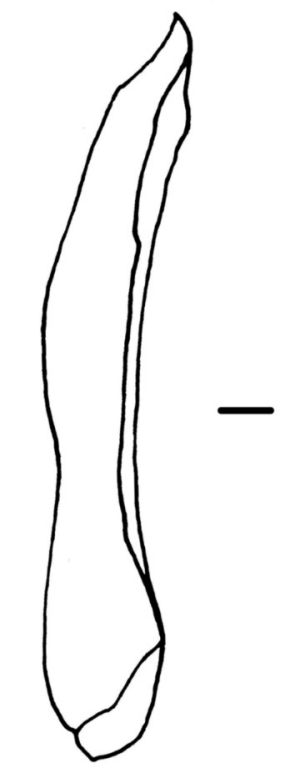

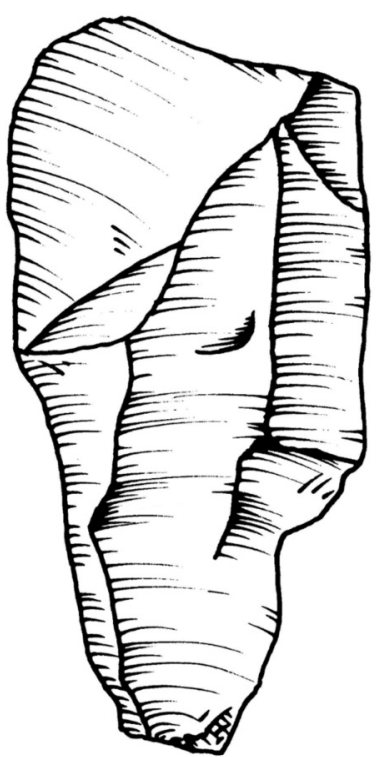

Planche 1 : Débitages unipolaires allongés. $A$ et $B$ : remontages de deux éclats à dos; $C$ et $D$ : éclats allongés unipolaires. Plate 1 - Elongated unipolar flaking. $A$ and $B$ : refittings of two baked-flakes. $C$ and $D:$ unipolar elongated flakes. 
Enfin, deux nucléus présentent une exploitation de quatre et cinq surfaces alternativement de débitage/plan de frappe, conférant au nucléus une morphologie finale quadrangulaire ou polyédrique (pl. 2). Les produits sont généralement courts, de morphologie quadrangulaire.

\section{Débitage sur éclat et ramification des chaînes opératoires}

Le débitage sur éclat est important puisqu'il totalise 23,8 \% des nucléus ( $n=10$; tabl. 6$)$. Ce débitage prend des formes variées avec un débitage Kombewa à la fois récurrent et linéal $(n=7)$ ainsi que du débitage sur face supérieure d'éclat $(n=2)$ voire sur les deux faces $(n=1)$.

Si le débitage de concept Kombewa est le plus représenté dans sa globalité $(70 \%, n=7)$, différentes méthodes sont employées.

Parmi les méthodes linéales, deux nucléus montrent le détachement d'un premier éclat Janus tandis qu'un second éclat est obtenu suivant le même axe de débitage. Ce débitage secondaire représente une forme de récurrence avec deux produits obtenus de caractères morphotechniques bien différenciés notamment en termes de morphologie en section.

Les méthodes Kombewa récurrentes ( $n=3$; pl. 3 ) correspondent à un débitage depuis la partie proximale et les bords (bord droit, bord gauche ou bilatérale). Deux catégories de produits sont obtenues : des produits minces allongés ou des produits courts quadrangulaires. Les plans de frappe sont systématiquement aménagés et le plan de détachement des produits est majoritairement sécant.

Les produits issus du débitage sur face inférieure d'éclat sont plutôt bien représentés par rapport au nombre de nucléus puisque 12 sont présents dans la série (pl. 3).

Le fort pourcentage de nucléus sur éclat sous-tend l'existence de chaînes opératoires ramifiées (Bourguignon, Faivre et Turq 2004). Aux nucléus sur faces inférieures et supérieures d'éclats s'ajoutent deux nucléus alternants dont le support est un éclat.

\section{Le débitage Levallois}

Même si le concept de débitage Levallois ne domine pas dans ce niveau, son emploi est attesté par des éclats ( $n=17)$ comme par quelques nucléus $(16,7 \% ; n=7$; tabl. 7 et pl. 4). Des modalités linéales et récurrentes ont pu être identifiées. Ces dernières témoignent majoritairement d'une exploitation unipolaire ou bipolaire.

Le débitage Levallois identifié peut se répartir en deux catégories : un débitage Levallois classique (Boëda 1986) et un typo-Levallois (fig. 5). Dans ce dernier, le volume du nucléus n'est ni configuré ni exploité dans son intégralité. Seule une partie du volume de la matrice est concernée par le débitage. Dans le cas du nucléus présenté en figure 5 par exemple, la plupart des critères définis par É. Boëda (Boëda 1986) sont présents : nucléus conçu en deux surfaces asymétriques sécantes se recoupant en un plan d'intersection (ici partiel), surfaces hiérarchisées, aménagement du plan de frappe, détachement des produits parallèle au plan d'intersection et percussion dure minérale.
Seul l'aménagement des convexités est ici minimisé. Toutefois, les deux enlèvements latéraux préfigurent la morphologie et rectifient les convexités pour le détachement de l'éclat central.

Dans l'autre cas, à savoir un débitage Levallois classique, l'ensemble de la structure volumétrique est intégré pour le débitage de telle sorte que bloc et nucléus ne font plus qu'un. Dans tous les cas une attention particulière peut être portée aux plans de frappe alors que cette surface elle-même peut être aménagée à minima. Elle est visible sur les nucléus et les éclats dont le talon est facetté ou dièdre.

\section{Autres méthodes de débitage}

Trois nucléus montrent une gestion alternante de deux surfaces de débitage sans qu'ils puissent être raccordés avec certitude au concept Discoïde. Les séquences sont courtes et les produits de nature différente. Deux de ces nucléus sont par ailleurs réalisés à partir d'éclats épais. Des blocs avec un ou deux enlèvements sont également présents. Ces blocs sont juste testés et l'arrêt du débitage est très probablement lié à la qualité médiocre des blocs sélectionnés.

\section{4 - Analyse techno-morpho-fonctionnelle des pièces confectionnées}

\subsection{1 - Le façonnage}

Les proportions dans l'assemblage des concepts de façonnage sont très faibles $(0,46 \%)$. Une seule pièce façonnée bifaciale (fig. 6) a été réalisée bien que des éclats caractéristiques de ce mode de production soient présents $(n=17)$. Cette pièce témoigne d'une exploitation sans doute mixte (débitage/façonnage ; Brenet et al. 2014) avec une phase de mise en forme du support qui peut également avoir eu un objectif productionnel à l'instar du Trifacial (Boëda 1991). Cette première phase a lieu successivement sur les deux faces du support, par des enlèvements unipolaires envahissants voire très envahissants. Du cortex subsiste sur les deux faces du support délimitant une zone proximale corticale. Puis, dans un second temps, une phase de retouche vient régulariser les tranchants de l'outil tandis qu'une autre vient régulariser la zone de contact préhensif supposé opposée au tranchant.

Deux unités potentiellement transformatives (UTF CT) peuvent être individualisées sur cette pièce. La première se trouve en position distale gauche et est courte $(21 \mathrm{~mm})$, convexe en plan, rectiligne en profil d'une angulation de $65^{\circ}$. Elle est bi-plane en section. La seconde unité est située en partie proximale et mésiale gauche. Elle est denticulée en plan, rectiligne en profil et de section biplane ou concavo-plane. Son extension est plus grande $(55 \mathrm{~mm})$ tandis que l'angulation varie entre $70^{\circ}$ et $80^{\circ}$. Cet angle assez ouvert rend cette unité bivalente, pouvant également correspondre à une zone de maintien possible.

La zone supposée préhensive (UTF CP/CR) correspond à la partie corticale en zone proximale ainsi qu'au bord droit. Une phase de régularisation concerne cette unité. 
A

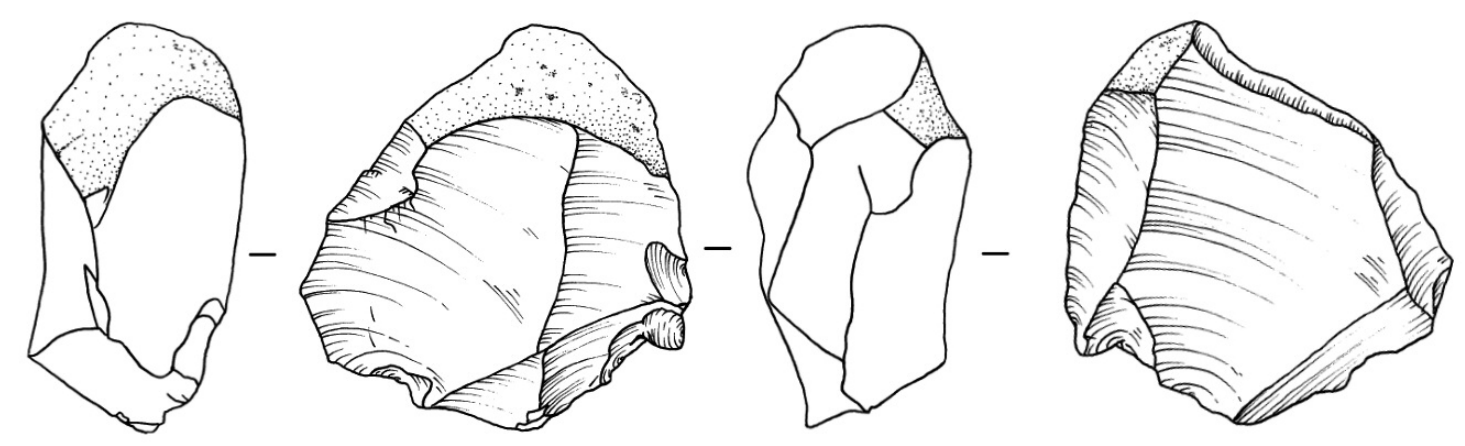

B
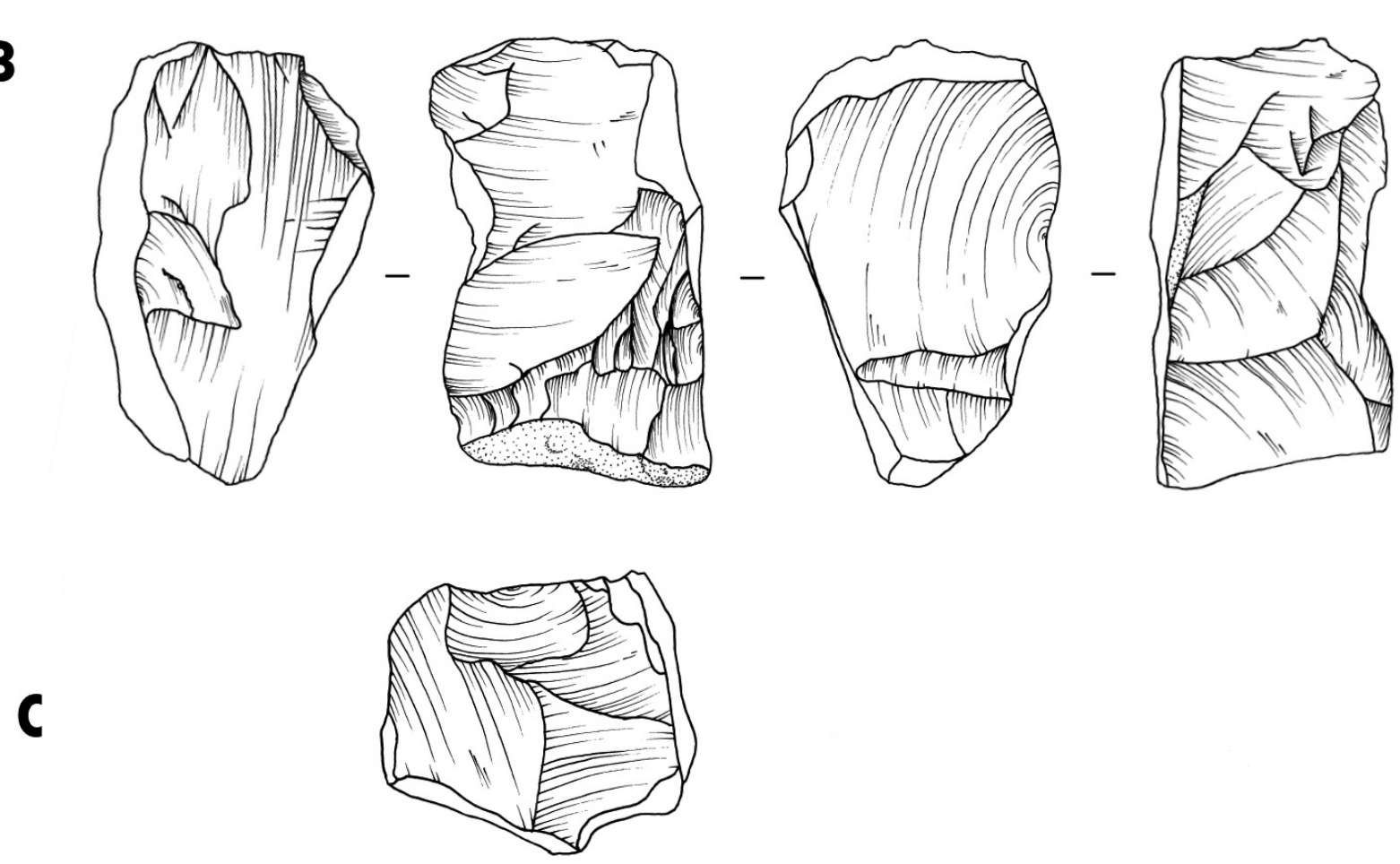

$$
\text { (1) }
$$
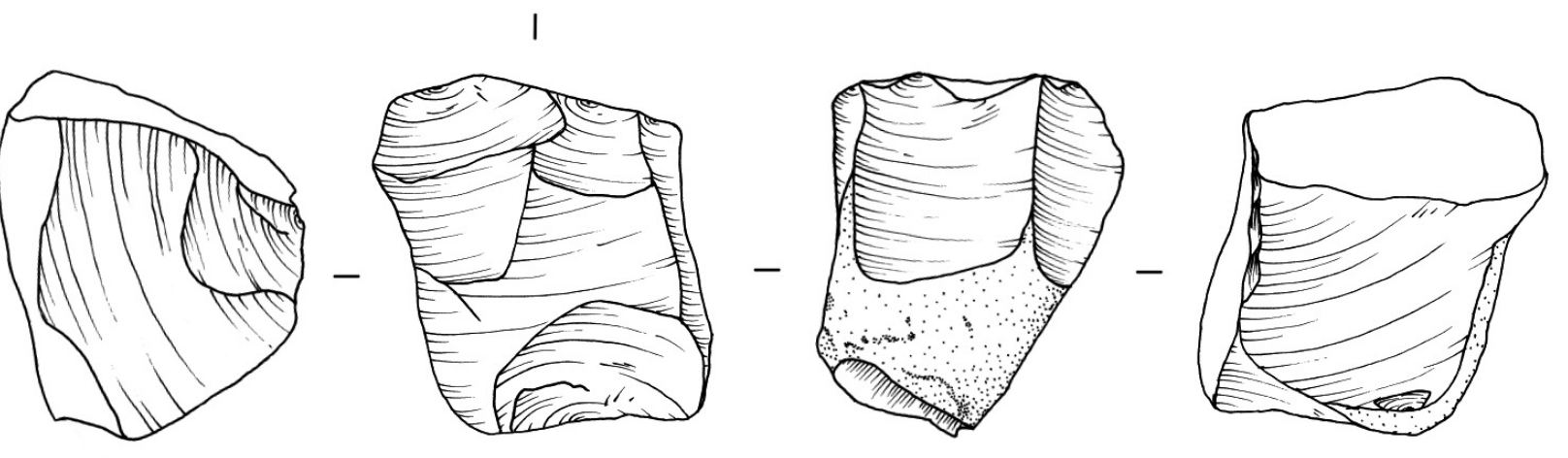

Planche 2 - Nucléus S.S.D.A. ; $A$ : à deux surfaces de débitage ; $B$ : à quatre surfaces de débitage ; $C$ : à cinq surfaces de débitage. Plate 2 - S.S.D.A. cores. A : two flaking surfaces ; $B$ : four flaking surfaces ; $C$ : five flaking surfaces. 


\begin{tabular}{lcc}
\hline \multicolumn{1}{c}{ Méthodes sur éclat } & Silex & $\%$ \\
\hline Kombewa linéal & 4 & $40,0 \%$ \\
Kombewa récurrent & 3 & $30,0 \%$ \\
Sur face inférieur et supérieure & 1 & $10,0 \%$ \\
Sur face supérieure & 2 & $20,0 \%$ \\
\hline Total & 10 & $100 \%$ \\
\hline
\end{tabular}

Tableau 6 - Méthodes de débitage sur éclats d'après les nucléus.

Table 6 - Cores on flake.

\begin{tabular}{lcc}
\hline \multicolumn{1}{c}{ Méthodes Levallois } & Silex & $\%$ \\
\hline Linéale (à pointe ou éclat) & 3 & $42,9 \%$ \\
Récurrente unipolaire & 1 & $14,3 \%$ \\
Récurrente bipolaire & 3 & $42,9 \%$ \\
\hline Total & $\mathbf{7}$ & $100 \%$ \\
\hline
\end{tabular}

Tableau 7 - Méthodes de débitage Levallois d'après les nucléus.

Tableau 7 - Levallois knapping following the cores.

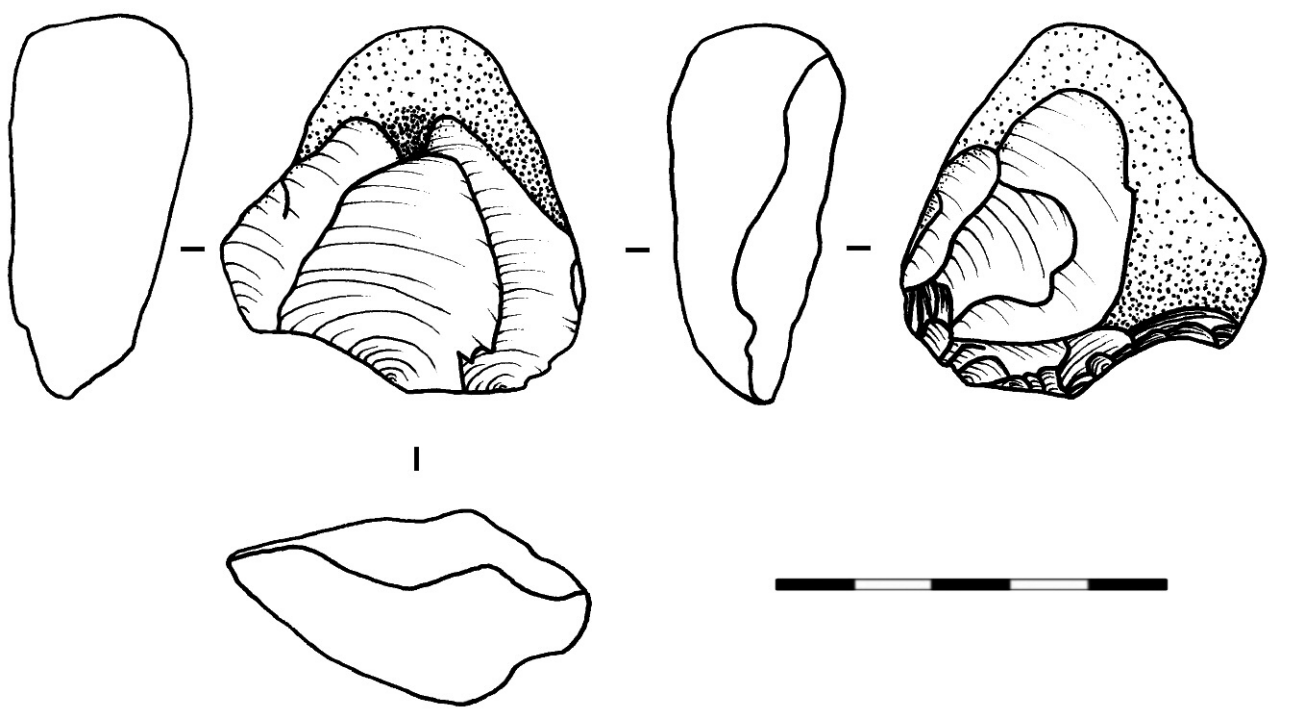

Figure 5 - Nucléus typoLevallois dont l'intégralité du volume n'est pas exploité, typo-Levallois.

Figure 5 - Levallois core with a partial exploitation of the volume, typo-Levallois.

\subsection{2 - Les outils sur supports débités}

$\mathrm{Au}$ sein de l'assemblage, 54 pièces présentent des retouches régulières (soit $1,38 \%$ du matériel total). Les pièces présentant des retouches pouvant être liées à des altérations taphonomiques (notamment les pièces à retouches abruptes alternes etc.) ont été écartées de l'étude. Quinze autres pièces ont été également éliminées de l'étude techno-morpho-fonctionnelle bien que la retouche soit intentionnelle. II s'agit d'une part, des pièces très altérées dont les angles des tranchants n'étaient pas mesurables et d'autre part, des fragments d'outils.

L'étude a donc porté sur 39 éclats retouchés (tabl. 8). D'un point de vue typologique, l'ensemble est dominé par les racloirs $(n=20 ; 51,28 \%)$ suivi du groupe des encoches et des denticulés ( $n=7 ; 17,95 \%)$. Les autres catégories d'outils sont des pointes, des grattoirs ainsi qu'une pièce dont le dos a été aménagé.

La retouche modifie peu les supports. Elle est majoritairement peu envahissante et ne concerne que rarement plus de la moitié de la surface de la pièce. Les éclats de retouche $(n=272)$, répartis en différents types (Bourguignon 1997) montrent une retouche qui se limite à un, deux voire trois rangs $(90,1 \%$ pour les types 0 , I et II). II s'agit en majorité d'éclats d'aménagement de racloirs, seuls sept éclats d'encoche ont été identifiés $(2,6 \%)$.

Les outils sont constitués en grande majorité d'une seule unité transformative potentielle $(n=29)$. II existe plusieurs catégories de tranchants : linéaires $(n=14)$, pointes $(n=6)$, bord-pointes $(n=4)$, bord-pointe-bords $(n=2)$ ou denticulés $(n=3)$ (tabl. 9). Sur certains supports on peut individualiser plusieurs unités $(n=10)$, qu'elles soient identiques (répétition de l'outil) ou différentes (complémentarité ou recherche fonctionnelle différente). Dans la plupart des cas deux unités sont associées. Seules trois pièces présentent trois unités associées. II existe également une pièce retouchée sur tout le pourtour pour laquelle la détermination des unités n'a pas été concluante (absence de régularité ou de rupture significative en termes en plan, profil, section ou angulation). Nous avons fait le choix de ne pas proposer 


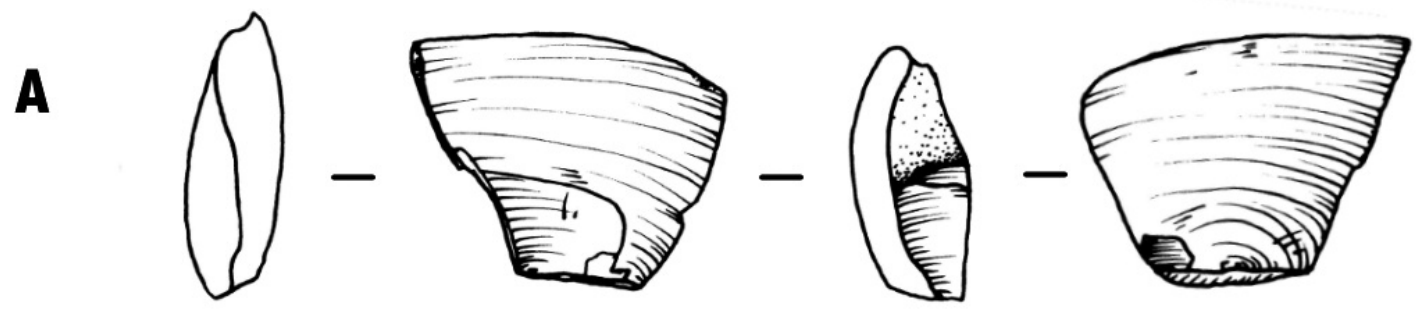

B

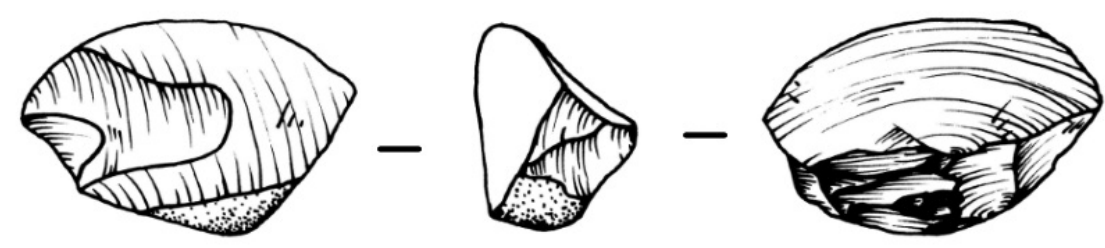

C
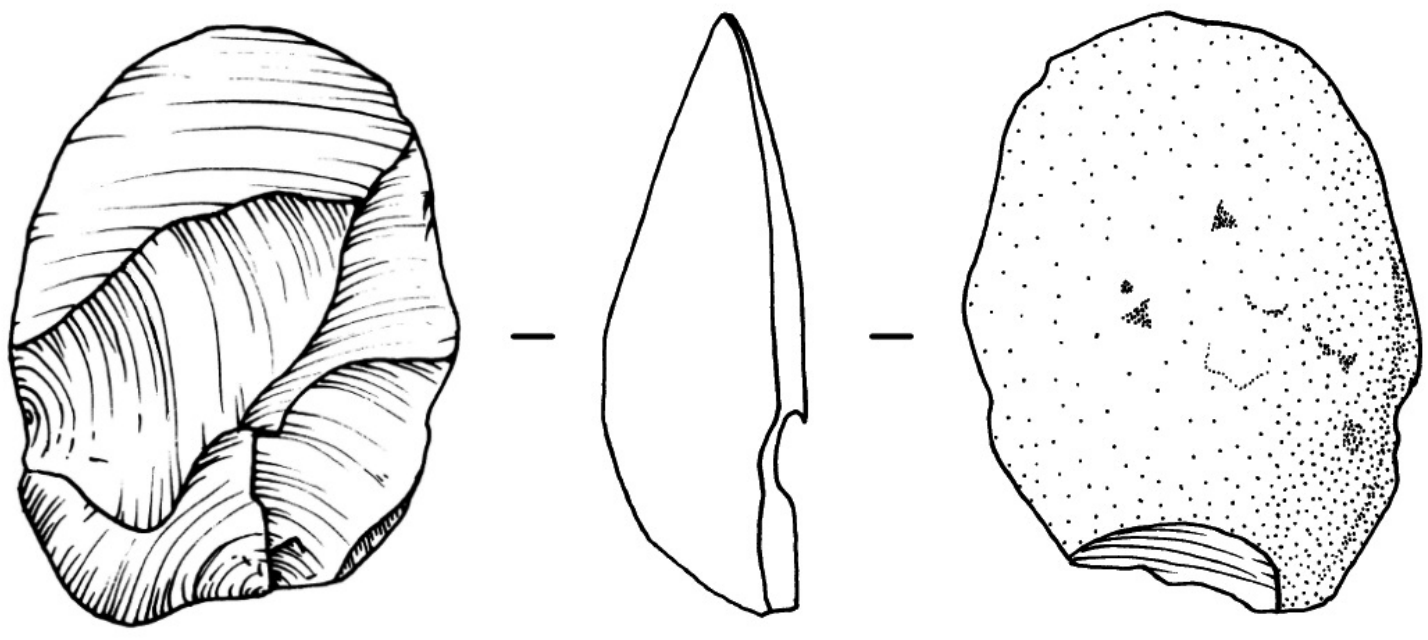

D
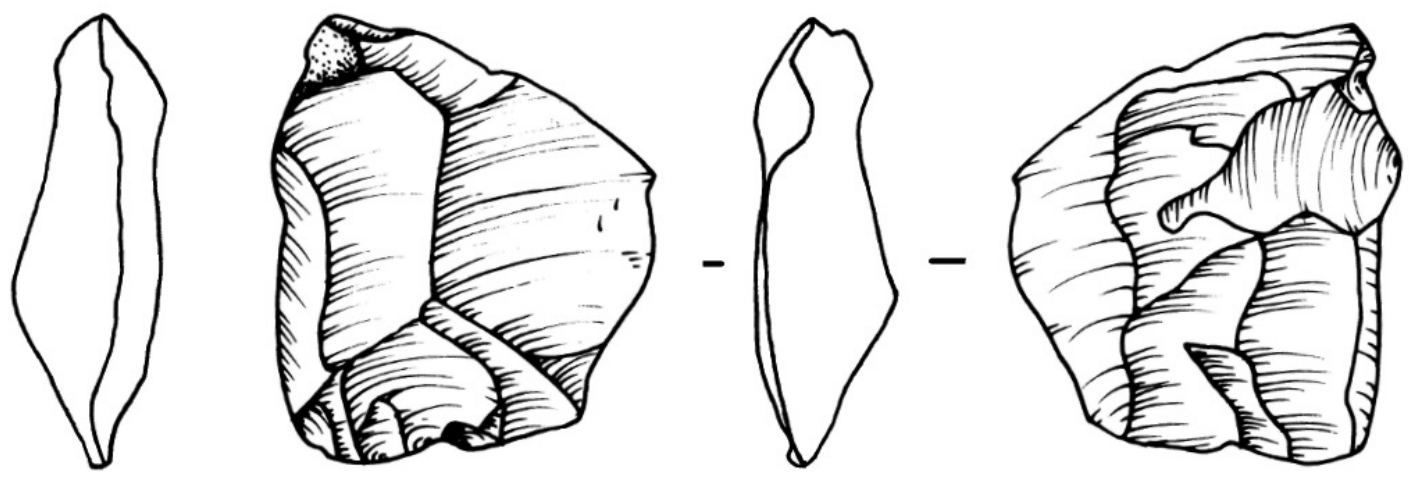

Planche 3 - Nucléus sur éclat et produits Kombewa. A et B : éclats Kombewa; $C$ et $D$ : nucléus sur éclat récurrents. Plate 3 - Cores on flakes and kombewa products. A and B : Kombewa flakes ; C and D : recurrent cores-on-flakes. 
A

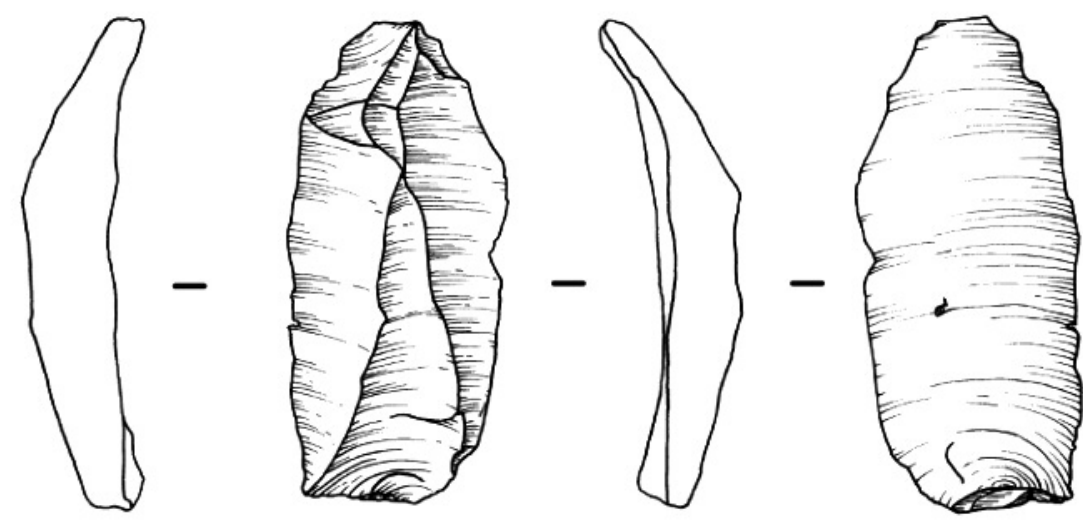

B
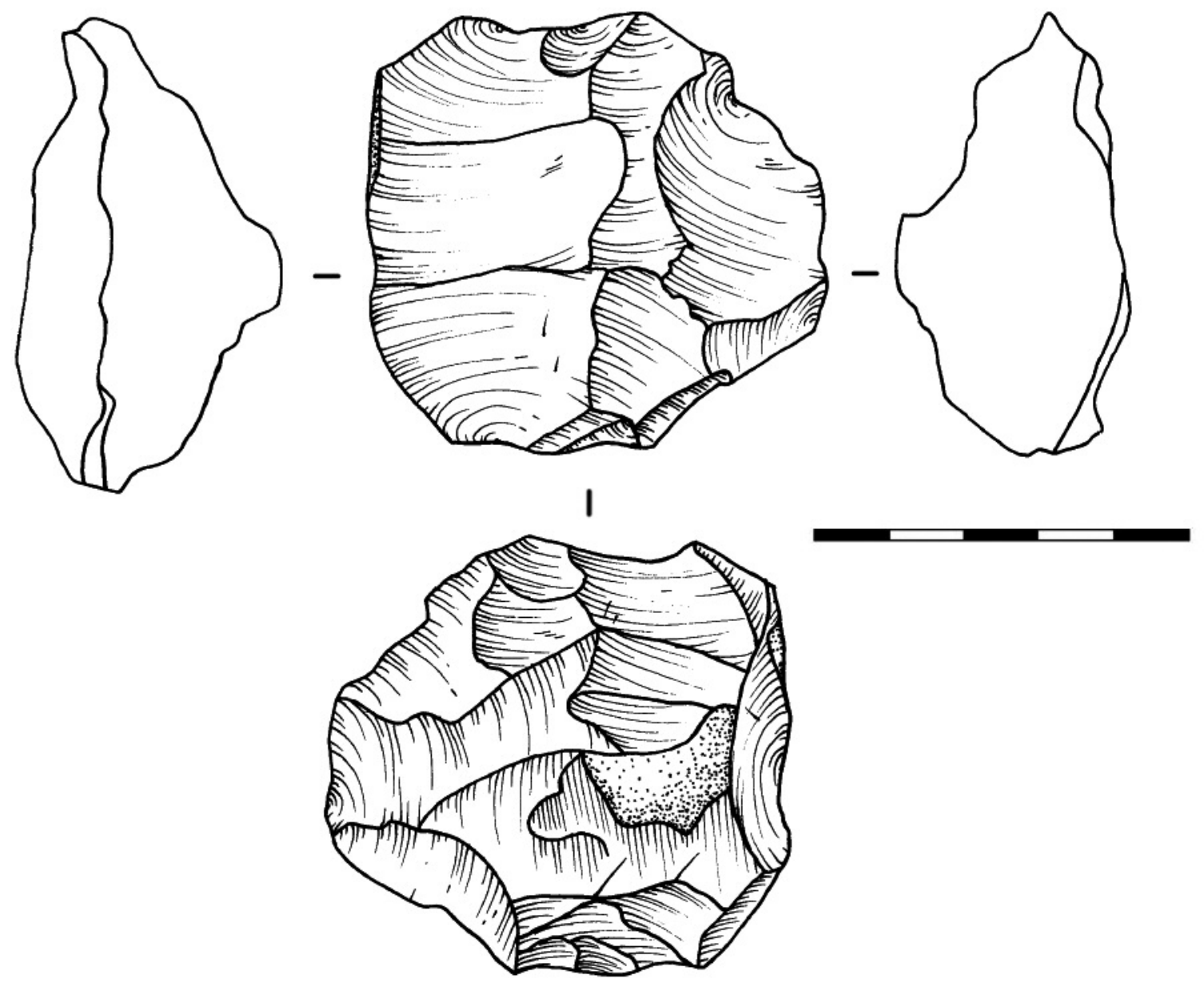

Planche 4 - Produit Levallois $(A)$ et nucléus (B).

Plate 4 - Levallois product $(A)$ and core (B). 


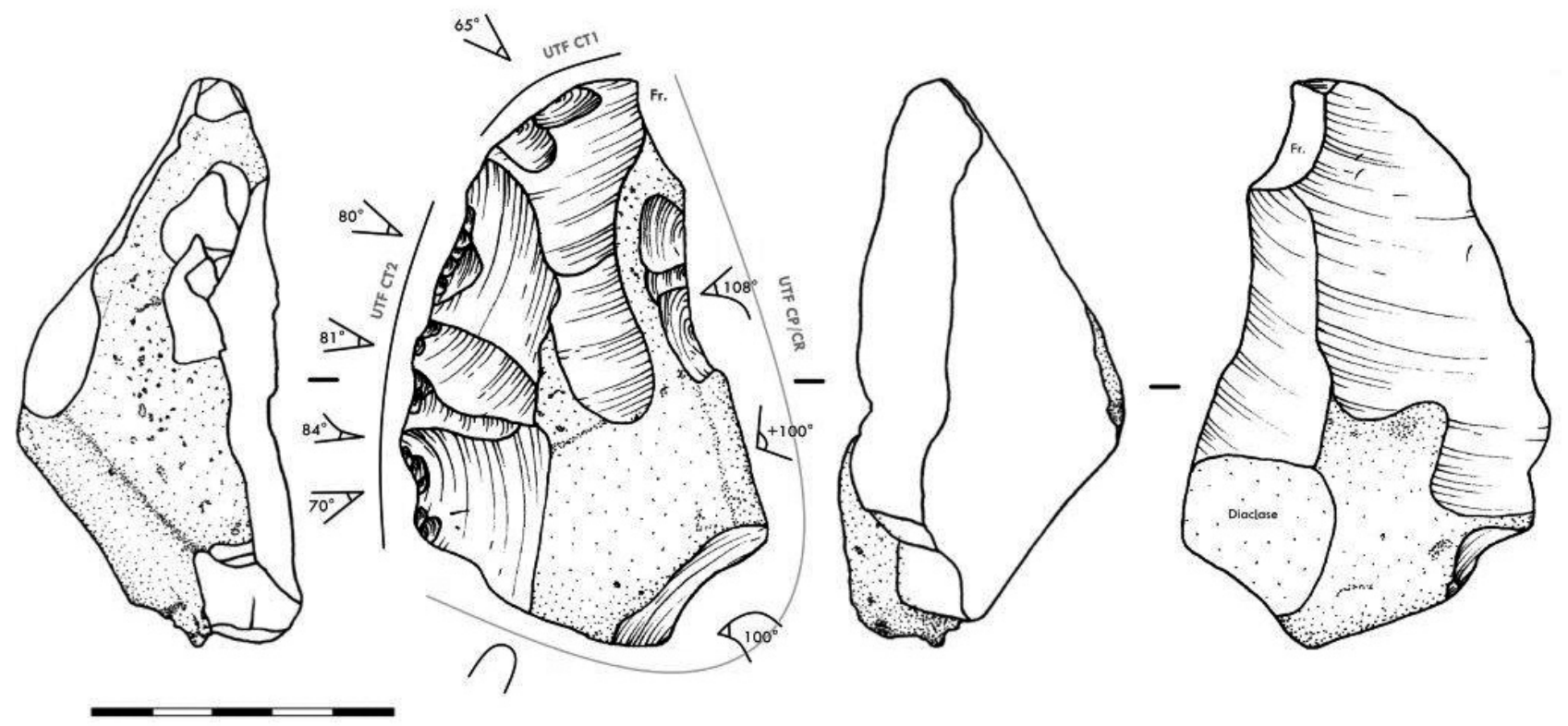

Figure 6 - Pièce bifaciale et unités identifiées.

Figure 6 - Bifacial tool and unities identified.

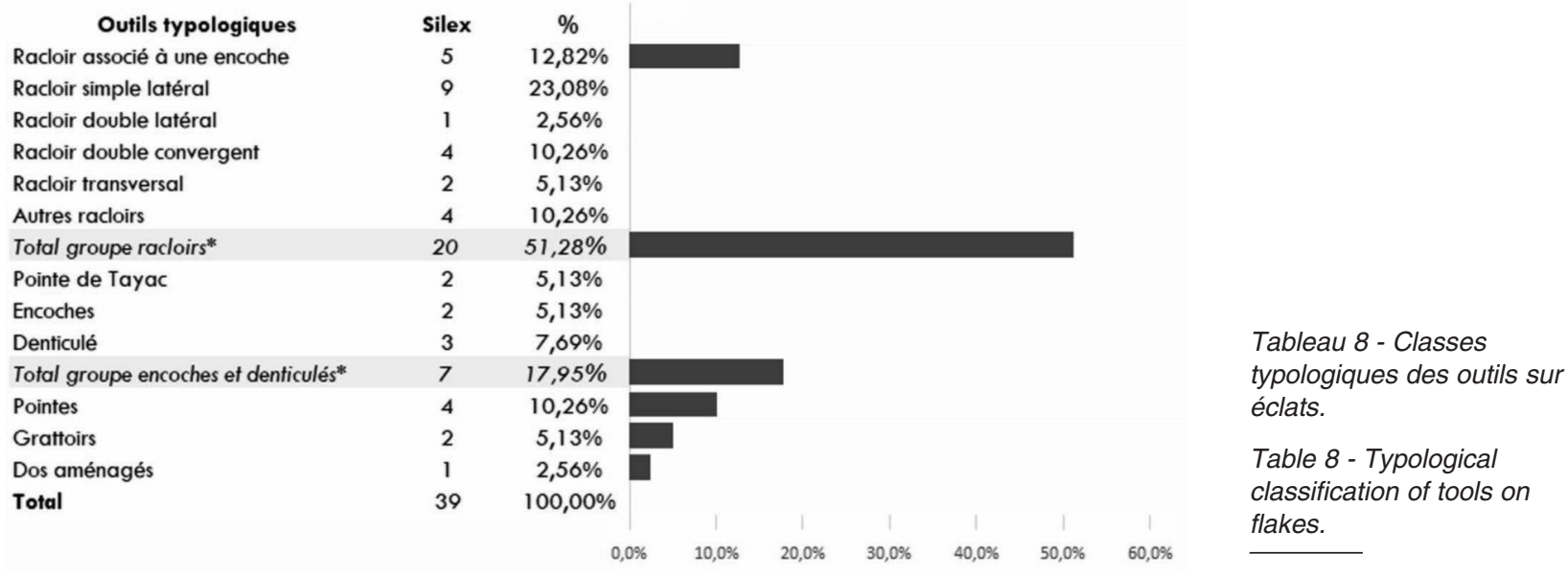

d'hypothèse quant à ses parties constitutives et ses orientations fonctionnelles.

\section{Les unités transformatives linéaires (UTF CT - Linéaires)}

II s'agit de la configuration la plus employée dans le niveau, avec 14 pièces pour les unités uniques auxquelles on peut ajouter 11 occurrences d'unités associées. Les zones de préhension (UTF CP) semblent être assez normalisées avec l'emploi majoritairement de supports à dos (corticaux, bruts de débitage).

Plusieurs catégories de contacts transformatifs peuvent être distinguées : rectilignes (pl. 5) et sinueuses (avec deux groupes d'angles) ainsi que convexes et concaves. À l'exception des tranchants sinueux, tous ces types se retrouvent dans les outils à unités transformatives multiples.

\section{Les unités transformatives punctiformes (UTF CT - Pointe ; Bord-Pointe et Bord-Pointe-Bord).}

Ces groupes sont très diversifiés et témoignent à chaque fois de peu pièces. Plusieurs catégories et sous-catégories peuvent être individualisées.

Pour les pointes (pl. 5), celles-ci pourront être rectilignes, mousses (de deux groupes d'angles) ainsi qu'en «bec » (deux encoches ou surfaces concaves se rejoignant en une pointe, sur la même face ou non). 


\begin{tabular}{|c|c|c|c|c|c|c|c|c|c|}
\hline \multirow[t]{2}{*}{ Types d'UTF CT } & \multicolumn{2}{|c|}{ N Silex } & \multirow[t]{2}{*}{$\%$} & \multirow[t]{2}{*}{ Angles } & \multirow[t]{2}{*}{ Médiane } & \multirow[t]{2}{*}{ Ecart type } & \multirow[t]{2}{*}{ Etendue } & \multirow[t]{2}{*}{ Médiane } & \multirow[t]{2}{*}{ Ecart type } \\
\hline & Uniques & Multiples & & & & & & & \\
\hline Linéaires & 14 & 11 & $51,0 \%$ & 68,04 & 68 & 19,8 & 29,3 & 25 & 10,8 \\
\hline Rectiligne angle $A$ & 3 & 3 & $12,2 \%$ & 47 & 45 & 10,3 & 28,8 & 23,5 & 14,7 \\
\hline Rectiligne angle $B$ & 4 & 3 & $14,3 \%$ & 82 & 84 & 7,5 & 31,1 & 25 & 11,2 \\
\hline Convexe & 2 & 3 & $10,2 \%$ & 68,2 & 80 & 24,3 & 27,6 & 25 & 5 \\
\hline Concave & 2 & 2 & $8,2 \%$ & 70,7 & 64 & 16,6 & 22 & 20 & 6,3 \\
\hline Sinueux angle $A$ & 2 & - & $4,1 \%$ & 60 & 60 & 7 & 44,5 & 44,5 & 3,5 \\
\hline Sinueux angle $B$ & 1 & - & $2,0 \%$ & 95 & - & - & 27 & - & - \\
\hline Denticulées & 3 & 3 & $12,2 \%$ & 80,8 & 82,5 & 7,3 & 42,3 & 35,5 & 15,1 \\
\hline Pointes & 6 & 3 & $18,4 \%$ & 77,5 & 85 & 19,5 & 20,3 & 22 & 5,5 \\
\hline Rectilignes & 3 & 1 & $8,2 \%$ & 85 & 85 & 4 & 19,2 & 19,5 & 7,6 \\
\hline Mousses angle $A$ & 1 & - & $2,0 \%$ & 38 & - & - & 24 & - & - \\
\hline Mousses angle $B$ & 2 & - & $4,1 \%$ & 90 & 90 & 0 & 17 & 17 & 1,4 \\
\hline Bec & - & 2 & $4,1 \%$ & 70 & 70 & 28,2 & 24 & 24 & 1,4 \\
\hline Bord-Pointes & 4 & 3 & $14,3 \%$ & 66,4 & 75 & 23 & 30,7 & 32 & 14,1 \\
\hline Rectiligne & 2 & 1 & $6,1 \%$ & 66 & 73 & 25 & 38,6 & 32 & 16 \\
\hline Association avec une fracture & 1 & - & $2,0 \%$ & 78 & - & - & 33 & - & - \\
\hline Denticulée & - & 1 & $2,0 \%$ & 90 & - & - & 14 & - & - \\
\hline Rectiligne/convexe & 1 & - & $2,0 \%$ & 60 & - & - & 32 & - & - \\
\hline Bord-Pointe-Bords & 2 & - & $4,1 \%$ & 85 & 85 & 7 & 68 & 68 & 25,4 \\
\hline Total & 29 & 20 & $100 \%$ & - & - & - & - & - & - \\
\hline
\end{tabular}

Tableau 9 - Types de tranchants identifiés sur les outils, angulation et étendue.

Table 9 - Edges identified on tools, angulation and entent.
Concernant les bord-pointes, les associations entre un bord et la pointe sont variées : rectilignes, pointe adossée à une surface de fracture (type «burin »), bord denticulé, ou encore bord rectiligne et pointe convexe. Théoriquement, bien d'autres associations sont possibles. Ici se pose la question de la complémentarité dans l'activé du bord associé à la pointe.

\section{Les unités transformatives denticulées}

Ce type de tranchant est assez peu représenté (trois unités uniques et trois associées). Une difficulté majeure subsiste quant à la zone véritablement recherchée dans l'unité : le creux de l'encoche (concavo-plan ou concavo-convexe, d'angulation élevée) ou le denticule/bec d'angle (biplan et dont l'angulation diffère entre 5 et $10^{\circ}$ ). Ainsi, des tranchants à la fois linéaires concaves et punctiformes peuvent être utilisés, ils peuvent être utilisés conjointement (de cette manière toutes les caractéristiques seraient recherchées dès le départ) ou indépendamment (un des deux tranchants serait alors simplement la conséquence du tranchant principalement recherché).

\section{Les unités transformatives multiples}

Comme nous l'avons vu, neuf supports présentent plusieurs unités transformatives. Au sein de ces outils se trouvent des unités qui ne sont pas matérialisées par une phase de confection (tranchants bruts). Ainsi, les unités associées retouchées peuvent parfois correspondre à des zones d'accommodation de la zone préhensive par leur caractère bivalent (pouvant à la fois être utilisées mais dont l'angulation permet le maintien) (pl. 5).

\section{3 - Discussion}

\section{Bilan des comportements techniques identifiés dans la couche $L 2 / 3$}

Ce niveau se caractérise par une production principale qui correspond à un débitage algorithmique unipolaire latéralisé et occasionnellement bipolaire visant à produire des supports normalisés, allongés et à dos. La sousreprésentation des nucléus associés à ce concept est à souligner. Pour l'expliquer, il est possible que les nucléus aient été exploités suivant d'autres objectifs de production, dénaturant ainsi le nucléus initial (gestions matricielles successives différenciées). Les nucléus pourraient également se trouver hors de la zone fouillée. L'introduction de produits allongés sur le site peut être envisagée bien qu'aucun élément ne permette d'étayer véritablement cette hypothèse. Les études récentes menées sur les productions laminaires et allongées du Sud-Ouest de la France montrent un séquençage important de ces chaînes opératoires (Ortega et al. 2013)

Associées à cela, des productions diversifiées existent. D'autres méthodes algorithmiques, sur éclats, Levallois ou encore alternantes sont présentes. Les supports produits sont tout aussi variés que les concepts de débitages qui y sont associés. Le concept Trifacial évoqué précédemment (Delpech et al. 1995) n'est pas présent dans la série.

Après étude il apparaît que l'outillage sur éclat soit très peu normalisé et ce, malgré le manque de diversité visible au travers des classes typologiques identifiées (50\% de racloirs). Si au sein des supports retouchés une recherche de pièces à tranchants linéaires semble prépondérante, dans le détail, ces derniers offrent des caractéristiques morphologiques très diversifiées avec six groupes identifiés. Une recherche de contacts punctiformes est aussi présente avec cinq groupes identifiés. Certaines pièces comprennent également des tranchants linéaires ainsi que des contacts punctiformes, répartis en cinq catégories.

Excepté pour les tranchants linéaires qui sont sur des supports de plein débitage, ce sont surtout des sous-produits des chaînes opératoires qui sont sélectionnés pour être retouchés : éclats corticaux, semi-corticaux et à cortex résiduel. 


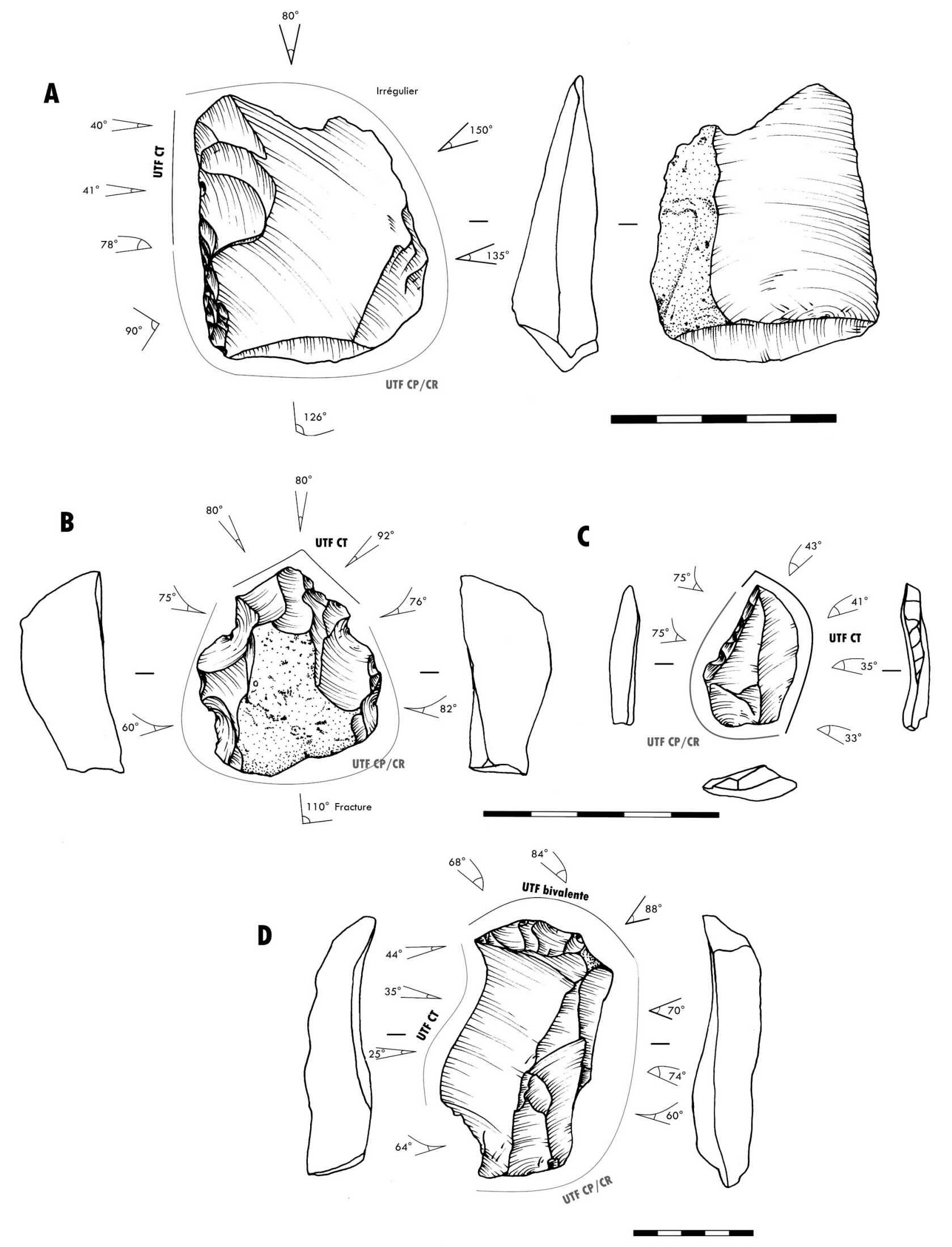

Planche 5 - Analyse techno-morpho-fonctionnelle des outils sur éclats. A : racloir simple latéral ; $B$ : denticulé convergent («Pointe de Tayac "); $C$ : dos aménagé ; $D$ : grattoir.

Plate 5 - Techno-morpho-functional analysis of retouched flake-tools. A : side-scraper ; B : denticulate (“Tayac point") ; : retouched back : $D$ : front-scraper. 
Si aucune normalisation n'est perceptible pour la confection de l'outillage, elle existe dans le mode de production de supports allongés à dos (majoritairement corticaux). Ces produits offrent des caractères morpho-techniques très proches avec un tranchant linéaire rectiligne opposé à un dos, souvent envahissant, parfois débordant. Ainsi, l'outil brut ne nécessiterait aucun aménagement a posteriori de sa zone préhensive puisque prévue et intégrée par le débitage. Quelques-uns de ces produits ont été utilisés pour la confection d'outils à contacts linéaires.

Le façonnage n'est représenté que par une pièce bifaciale de petites dimensions qui peut également être pourvoyeuse d'éclats, eux même potentiellement sélectionnés pour être utilisés.

Parmi les supports disponibles, peu sont sélectionnés parmi le mode de production principal (unipolaire asymétrique) pour être confectionnés. Dans le cas des contacts linéaires, la retouche n'est utilisée que pour régulariser le tranchant ou le délimiter, ou bien pour accommoder probablement une zone de maintien opposée au contact linéaire laissé brut (comme c'est le cas des pièces présentant plusieurs unités transformatives potentielles associées). À l'inverse, la retouche a un véritable rôle dans la mise en place des contacts punctiformes (pointe, bord-pointe et bord-pointe-bord), pour lesquels il est difficile d'obtenir ces morphologies uniquement par débitage.

\section{La couche L2/3 dans son contexte régional}

Ainsi, du point de vue de la composition de la série, de ses traits technologiques ainsi que de sa position chronologique, ce niveau de la Micoque s'intègre parfaitement parmi les premières manifestations du Paléolithique moyen aquitain tel que défini actuellement. Chronologiquement, c'est l'industrie du niveau 2 de Petit-Bost (MIS 9/8) qui serait à rapprocher de ce niveau. Technologiquement, on note cependant un certain nombre de différences. À Petit-Bost, le façonnage bifacial est plus fréquent et une production trifaciale est présente (Bourguignon et al. 2008 ; Mathias et Bourguignon en préparation). Le débitage Levallois est plus représenté et un débitage Quina a également été identifié (Bourguignon et al. 2008). En ce qui concerne les traits communs, outre la présence du Levallois, une production algorithmique unipolaire, ainsi que des chaînes opératoires ramifiées, sont employées.

À partir du MIS 8 et jusqu'à la fin du MIS 6, l'utilisation du débitage Levallois semble se généraliser au sein des industries de la région même si elle est parfois minoritaire voire absente de certains sites. En dehors du débitage Levallois, de nombreux autres concepts sont utilisés : Bifacial, Trifacial, Discoïde, Quina, Laminaire et algorithmiques s.l.. L'Aquitaine montre donc des indices d'une grande diversité technique, notamment aux MIS 7/6 (Brenet et al. 2014).

Si on ne considère que la production d'éclats allongés à dos par des méthodes unipolaires, dans le Paléolithique moyen ancien local elle est présente dès le MIS 9 dans le niveau 2 de Petit-Bost (Bourguignon et al. 2008) ou plus récemment au MIS 6 à Combe-Brune 2 (Brenet 2011). Si la production d'outils bifaciaux dans ce niveau de la Micoque est anecdotique (une seule pièce façonnée et quelques éclats), la persistance d'une tradition bifaciale en Aquitaine à des périodes plus récentes est avérée: niveau 6 de La Micoque (Rosendahl 2006) ou encore au MIS 6 dans le Bergeracois de Barbas C'3 base (Boëda et al. 1996 ; Boëda 2001) ou de Combe-Brune 2 (Brenet 2011). Pour les trois derniers sites mentionnés, le caractère très spécifique des industries du Bergeracois peut être évoqué (qualité et modules du silex maastrichtien). II n'en est pas de même pour la Micoque où les ressources minérales disponibles sont identiques à celles des autres niveaux. Cette persistance de traditions « acheuléennes » n'est pas un cas unique dans le Sud de la France puisqu'on la retrouve par exemple dans les Alpes-Maritimes, sur le site du Lazaret daté du MIS 6 (Lumley et al. 2004).

La présence conjointe de séries avec le « tout éclat » et de séries utilisant le façonnage bifacial ouvre le débat sur l'existence possible de deux traditions culturelles différentes, de la coexistence envisageable de plusieurs groupes sur un territoire restreint au cours d'une période relativement longue ou du rôle des activités menées ainsi que du type de site (occupations de plein-air, occupations en grottes) ou encore de sa fonction dans le territoire. La diversité de la composition des assemblages lithiques régionaux pourrait être le témoignage des différents contacts entre groupes distincts ou à l'inverse le témoignage de résistances culturelles (maintien de comportements quels que soient les échanges), pouvant varier au cours de cette période en fonction des systèmes de mobilités de ces groupes. Ceci pourrait expliquer la grande diversité technique que l'on rencontre dans ces industries du Paléolithique moyen ancien. Les comportements techno-économiques de cette période en Aquitaine sont d'ailleurs considérés comme préfigurant la complexité du Paléolithique moyen classique (Brenet et al. 2014).

\section{Et à l'échelle du Sud de la France?}

À une échelle plus large, la moitié sud de la France livre des sites du Paléolithique moyen ancien à débitage Levallois : en Ardèche, à Orgnac 3 au MIS 9/8 dans les niveaux 3, 2 et 1 (Combier 1967 ; Moncel 1999 ; Moncel et al. 2012), dans le Lot sur le site des Bosses (Jarry et al. 2007) ou encore au MIS 8 à la Baume-Bonne (Gagnepain et Gaillard 2003). Ces débitages sont systématiquement associés à quelques pièces bifaciales. Les proportions du concept Levallois ne sont toutefois pas les mêmes que dans la couche L2/3 de La Micoque où le débitage Levallois reste faiblement utilisé.

Dans la vallée du Rhône, où le débitage Levallois apparaît de façon précoce et ramifiée dans les niveaux supérieurs d'Orgnac 3 (Moncel 1999 ; Mathias 2016), il existe une diversité d'industries qui semble assez proche de celle du Paléolithique moyen ancien aquitain dans un contexte où le silex est également utilisé quasi-exclusivement. Ainsi au MIS 7 à Payre (Moncel 2008 ; Carmignani et al. 2017), des 
pièces bifaciales sont associées à un débitage Discoïde et orthogonal, tandis que sur l'autre rive du Rhône, au Bau de l'Aubesier toujours au MIS 7, un débitage Levallois récurrent centripète est associé à un débitage laminaire volumétrique, sans pièces bifaciales (Carmignani et al. 2017).

Des industries particulières ont été définies dans le bassin de la Garonne à des périodes similaires. L'Acheuléen Pyrénéo-Garonnais se caractérise ainsi par la production de grands supports associés notamment à des hachereaux (Mourre et Colonge 2007 ; Turq et al. 2010).

L'Acheuléen Pyrénéo-Garonnais et le Paléolithique moyen ancien de cette région pourraient se développer en parallèle à l'instar de ce que l'on connaît en Dordogne entre le MIS $9 / 8$ et le MIS 6 pour les sites de Duclos et Romentères (Hernandez et al. 2012).

\section{Quelles explications à cette diversité ?}

Plusieurs raisons peuvent être à l'origine de la diversité des assemblages lithiques du Paléolithique moyen ancien. Ces éléments sont les mêmes que ceux évoqués pour expliquer les différents faciès du Moustérien : environnementaux et climatiques (Rolland 1988), chronologiques (Mellars 1989), fonctionnels, systèmes de mobilités (Binford et Binford 1966), degré d'utilisation des outils et ravivages éventuels (Dibble 1984) ou encore traditions culturelles (Bordes et Bourgon 1951).

Entre le MIS 10 et le MIS 6, nous disposons de données inégales, concernant notamment les comportements de subsistance avec l'absence de restes fauniques sur les sites de plein-air. De la même façon, nous disposons de trop peu de ce type de sites pour percevoir des différences entre ces occupations (La Micoque, Petit-Bost et plus récemment les sites du Bergeracois) et celles en grotte (Vaufrey, Pech de l'Azé II). Les matières premières peuvent influencer les assemblages, ainsi des différences entre les sites du Bergeracois et ceux du Sarladais ou de la vallée de la Vézère sont clairement visibles. Malgré cela, une grande variabilité existe au sein même de ces zones, rendant caduque ce déterminisme minéral (Boëda et al. 1996 ; Brenet et al. 2008, 2014 ; Turq et al. 2017).

$\mathrm{Au}$ vu des données archéologiques dont nous disposons, aucune hypothèse n'explique entièrement cette variabilité. La diversité observée au Paléolithique moyen ancien, résultat de phénomènes multifactoriels, préfigure assurément la complexité du Moustérien des MIS 5 à 3.

\section{Conclusions}

Les systèmes techniques identifiés dans la couche L2/3 de La Micoque témoignent d'une production orientée vers des supports à dos, obtenus par des méthodes algorithmiques unipolaires ou bipolaires. Autour de ces méthodes gravitent une grande variété d'autres concepts de production, parmi lesquels des méthodes de débitage sur éclats ou Levallois. L'utilisation du débitage Levallois, la présence de chaînes opératoires ramifiées, une normalisation des produits, sont autant d'éléments caractérisant ce Paléolithique moyen ancien daté du MIS 10/9. Le débitage Levallois, bien que souvent minoritaire au sein de l'industrie, a donc été utilisé dès cette date dans la vallée de la Vézère. II est employé sans équivoque à partir du MIS 9 dans la région entre autre dans les niveaux 1 et 2 de Petit-Bost (MIS 9 et MIS 8/7) où il est associé à d'autres concepts de débitage et de façonnage (Bourguignon et al. 2008). La particularité de ce niveau de La Micoque réside avant tout dans les proportions des différents types de débitages employés. La part des produits confectionnés, tout en étant diversifiée, est faible $(1,38 \%)$, et ne présente qu'une unique pièce bifaciale Ce sont surtout des tranchants linéaires bruts qui sont présents en grande quantité, avec un dos opposé et issus du principal mode de production algorithmique.

Du point de vue des concepts de débitages utilisés, l'assemblage de la couche L2/3 de La Micoque s'intègre parfaitement au sein de la variabilité identifiée dans le Paléolithique moyen ancien d'Aquitaine et plus largement d'Europe de l'Ouest.

\section{Remerciements}

Nous tenons à remercier Jean-Jacques Cleyet-Merle pour l'accès aux collections des fouilles récentes de La Micoque ainsi que l'équipe du MNP, Peggy Bonnet-Jacquement, Bernard Nicolas, Stéphane Madelaine, Catherine Cretin, Marie-Dominique Dehé, Alain Turq et André Morala pour leur aide et leur accueil chaleureux. Jean-Philippe Rigaud a accueilli cette étude avec enthousiasme et a mis à disposition son fond documentaire au musée, nous l'en remercions. Merci à Laurence Bourguignon et Cyril Viallet pour les relectures attentives et leurs nombreux conseils ainsi qu'à Christophe Falguères pour avoir répondu à nos questions. Nous tenons également à remercier Jean-Pierre Texier pour ses précisions et éclaircissements sur les modalités de dépôt et le cadre chronologique, ainsi que deux rapporteurs anonymes dont les remarques ont permis d'enrichir le manuscrit. Cette étude, réalisée dans le cadre d'un travail doctoral, a bénéficié du soutien financier de l'Université de Perpignan Via-Domitia, de l'UMR 7194 HNHP ainsi que de l'ED 544 Inter-Med.

\section{Références bibliographiques}

ASHTON N. 1992 - The High Lodge flint industries. In : Ashton N., Cook J., Lewis S.J., Rose J. (Eds.), High Lodge. Excavations by G. de Sieveking, 1962-8, and J. Cook, 1988. British Museum Press, p. 124-168.

BINFORD L., BINFORD S. 1966 - Preliminary analysis of functional variability in the Mousterian of Levallois facies. American Anthropologist, t. 68, n², 2, p. 238-295.

BOËDA E. 1986 - Approche du concept Levallois et évaluation de son champs d'application à travers trois gisements saaliens et weichséliens de la France septentrionale. Paris : Université Paris X-Nanterre,Thèse de Doctorat, $181 \mathrm{p}$.

BOËDA E. 1991 - La conception trifaciale d'un nouveau mode de taille préhistorique. In : Bonifay E., Vandermeersch B. (Dir.). Les premiers européens. Actes 
du $114^{\circ}$ congrès des sociétés savantes, Comité des travaux historiques et scientifiques, p. 251-263.

BOËDA E. 1993 - Le débitage discoïde et le débitage Levallois récurrent centripète. Bulletin de la Société Préhistorique Française, t. 90, 6, p. 392-404.

BOËDA E. 1997 - Technogenèse de systèmes de production lithique au Paléolithique inférieur et moyen en Europe occidentale et au Proche-Orient. Paris : Université Paris X-Nanterre, 1997, Habilitation à Diriger des Recherches, $173 \mathrm{p}$.

BOËDA E. 2001 - Détermination des unités technofonctionnelles de pièces bifaciales provenant de la couche acheuléenne C'3 base du site de Barbas I. In : D. Cliquet (Dir.), Les industries à outils bifaciaux au Paléolithique moyen d'Europe occidentale. Actes de la table ronde internationale, Caen (Basse-Normandie, France) le 1415 octobre 1999, Liège, ERAUL 98, p. 51-76.

BOËDA E. 2013 - Techno-logique et technologie. Une paléo-histoire des objets lithiques tranchants. Archéoéditions, $266 \mathrm{p}$.

BOËDA E., KERZAVO B. 1991 - Une vieille industrie du Sud-Ouest de la France : le niveau inférieur de Barbas (Dordogne). In : Bonifay E., Vandermeersch B. (Dir.) Les premiers Européens, Actes du $114^{\mathrm{e}}$ congrès national des sociétés savantes, Paris, 1989, p. 27-38.

BOËDA E., KERZAVO B., MERCIER N., VALLADAS H. 1996 - Barbas C'3 base (Dordogne). Une industrie bifaciale contemporaine des industries du Moustérien ancien : une variabilité attendue. In: Bietti A., Grimaldi S. (Eds.), Reduction Processes ("chaînes opératoires") for the European Mousterian, Actes Du Colloque International de Rome. Quaternaria nova, VI, Rome, may 26-28 1995, p. $465 \mathrm{e} 504$.

BORDES F. 1950 - L'évolution buissonnante des industries en Europe occidentale : considérations théoriques sur le Paléolithique ancien et moyen. L'Anthropologie, t. 54, p. 393-420.

BORDES F. 1969 - Livret-guide de l'excursion A5 : Landes-Périgord. Union Internationale pour l'Etude du Quaternaire (INQUA), VII Congress, Paris, p. 44-47.

BORDES F. 1971 - Observations sur l'Acheuléen des grottes en Dordogne. Munibe, vol. 23, 1, p. 5-23.

BORDES F. 1984 - Leçons sur le Paléolithique. In: Le Paléolithique en Europe, tome II. CNRS Editions, ed. Cahiers du Quaternaire, 7, Paris.

BORDES F., BOURGON M. 1951 - Le complexe moustérien : moustériens, levalloisien et tayacien. L'Anthropologie, $n^{\circ} 55$, p. 1-23.

BOURGUIGNON L. 1997 - Le Moustérien de type Quina : nouvelle définition d'une entité technique. Paris : Université de Paris X-Nanterre, Thèse de Doctorat, 2 tomes, 672 p.
BOURGUIGNON L., DJEMA H., BERTRAN P., LAHAYE C., GUIBERT P. 2008 - Le gisement Saalien de Petit-Bost (Neuvic, Dordogne) à l'origine du Moustérien d'Aquitaine? In : Jaubert J., Bordes J.-G., Ortega I. (Dir.) Les sociétés du Paléolithique dans un grand sud-ouest de la France: nouveaux gisements, nouveaux résultats, nouvelles méthodes. Actes de la séance SPF, université de Bordeaux 1, Talence, 2006, Société préhistorique française, mémoire 47, p. 41-55.

BOURGUIGNON L., FAIVRE J-Ph., TURQ A. 2004 Ramification des chaînes opératoires : une spécificité du Moustérien ? Paleo, $n^{\circ}$ 16, p. 41-61.

BRENET M. 2011 - Variabilité et signification des productions lithiques au Paléolithique moyen ancien. L'exemple de trois gisements de plein-air du Bergeracois. Bordeaux : Université de Bordeaux I, 2011, Thèse de Doctorat, $480 \mathrm{p}$.

BRENET M., FOLGADO M., BERTRAN P., LENOBLE A., GUIBERT P., VIEILLEVIGNE E. 2008 - Interprétation de la variabilité technologique de deux industries du Paléolithique moyen ancien du Bergeracois : Cantalouette 1 et Combe Brune 3 (Creysse, Dordogne). Contexte géoarchéologique et chronologique, analyse technoéconomique. In : Jaubert J., Bordes J.-G., Ortega I. (Dir.) Les sociétés du Paléolithique dans un grand Sud-Ouest de la France : nouveaux gisements, nouveaux résultats, nouvelles méthodes, actes de la séance SPF, université de Bordeaux 1, Talence, 2006, Société préhistorique française, mémoire 47, p. 57-81.

BRENET M., BOURGUIGNON L., COLONGE D., FOLGADO M., JARRY M., LELOUVIER L.A., MOURRE V., TURQ A. 2014 - Les technocomplexes au début du Paléolithique moyen en Aquitaine septentrionale : complexité, complémentarité des productions de débitage et de façonnage et implications comportementales. In : Brenet M., Bourguignon L., Jarry M. (Eds.), Emergence et Diversité Des Techno-Complexes Du Paléolithique Moyen Ancien. Relations Entre Productions de Débitage et de Façonnage, Session C Du XXVIle Congrès Préhistorique de France, 31 Mai, 5 Juin 2010. Société Préhistorique Française, Bordeaux, Les Eyzies, p. 81-102.

BREUIL H. 1932 - Le Paléolithique ancien en Europe occidentale et sa chronologie. Bulletin de la Société préhistorique française, t. 29 , n. 12, p. 570-578.

CAPITAN L. 1896 - Station acheuléenne de La Micoque. Revue de l'Ecole d'anthropologie, p. 406-416.

CARMIGNANI L., MONCEL M.-H., FERNANDES P., WILSON L. 2017 - Technological variability during the early Middle Palaeolithic in Western Europe. Reduction systems and predetermined products at the Bau de l'Aubesier and Payre (South-East France). PLoS ONE, 12, 6, 49 p.

CHAUVET G., RIVIÈRE E. 1896 - Gisement quaternaire de la Micoque. Compte rendu de l'Académie des sciences, 24 août 1896, p. 284. 
CHEVRIER B. 2006 - De l'Acheuléen méridional au technocomplexe trifacial : la face cachée des industries du Bergeracois. Apport de l'analyse technologique de l'industrie lithique de Barbas I C'4 sup (Creysse, Dordogne). Gallia Préhistoire, vol. 48, p. 207-252.

COLONGE D., JARRY M., DELFOUR G., FONDEVILLE C., ARNOUX T., BERTHET A.-L. 2010 - De la transition paléolithique inférieur-moyen dans la vallée de la Garonne : l'Acheuléen supérieur de Raspide 2 (Blagnac, HauteGaronne). Bulletin de la Société préhistorique française, vol. $107, n^{\circ} 2$, p. 205-225.

COMBIER J. 1967 - Le Paléolithique de l'Ardèche dans son cadre paléoclimatique. Publication de l'Institut de Préhistoire de Bordeaux, Mémoire $n^{\circ} 4,462 p$.

DEBÉNATH A., RIGAUD J.-Ph. 1986 - Le gisement de La Micoque. In : Rigaud J.-Ph. (Dir.), Informations archéologiques: circonscription d'Aquitaine ; Gallia Préhistoire, 29, p. 236-237.

DEBÉNATH A., DELPECH F., GENESTE J.M., RIGAUD J.-Ph., TEXIER J.P. 1991 - Nouvelles recherches à La Micoque, Dordogne (France). In : Résumés du colloque commémoratif international de Miskolc (Hongrie). $5 \mathrm{p}$.

DELPECH F., GENESTE J.-M., RIGAUD J.-Ph. TEXIER J.-P. 1995 - Les industries antérieures à la dernière glaciation en Aquitaine septentrionale : chronologie, paléoenvironnements, technologie, typologie et économie de subsistance. Paleo, supplément, p. 133-163.

DIBBLE H. 1984 - Interpreting typological variation of Middle Palaeolithic scrapers : function, style or sequence reduction ? Journal of Field Archaeology, vol. 11, $\mathrm{n}^{\circ} 4$, p. 431-436.

FAIVRE J.-Ph. 2008 - Organisation techno-économique des systèmes de production dans le Paléolithique moyen récent du Nord-est Aquitain : Combe-Grenal et les Fieux. Paris : Université de Bordeaux I, Thèse de Doctorat , $477 \mathrm{p}$.

FALGUĖRES C., BAHAIN J.-J., SALEKI H. 1997 - USeries and ESR Dating of Teeth from Acheulian and Mousterian Levels of La Micoque (Dordogne, France). Journal of Archaeological Science, 24, p. 537-545.

FORESTIER H. 1992 - Approche technologique de quelques séries dites clactoniennes du Nord-ouest de la France et du Sud-est de l'Angleterre. Paris : Université Paris X-Nanterre, Mémoire de Maîtrise, $271 \mathrm{p}$.

FORESTIER H. 1993 - Le Clactonien : mise en application d'une nouvelle méthode de débitage s'inscrivant dans la variabilité des systèmes de production lithique du Paléolithique ancien. Paleo, n5, pp.53-82.

FROUIN M., LAHAYE C., HERNANDEZ M., MERCIER N., GUIBERT P., BRENET M., FOLGADO-LOPEZ M., BERTRAN P. 2014 - Chronology of the Middle Palaeolithic open-air site of Combe Brune 2 (Dordogne, France): a multi luminescence dating approach. Journal of Archaeological Science, 52, p. 524-534.

GAGNEPAIN J. et GAILLARD C. 2003 - La grotte de la baume Bonne (Quinson, Alpes de Haute-Provence) : synthèse chronostratigraphique et séquence culturelle d'après les fouilles récentes (1988-1997). John and Erica Hedges Ltd, p. 73-86.

GENESTE J.-M., PLISSON H. 1996 - Production et utilisation de l'outillage lithique dans le Moustérien du Sudouest de la France : les Tares à Sourzac, vallée de l'Isle, Dordogne. In : A. Bietti, S. Grimaldi (Eds.), Reduction Processes (" chaînes opératoires ») for the European Mousterian (Rome, 26-28 mai 1995). Actes de la table ronde internationale, Quaternaria nova, $n^{\circ}$ 6, p. 343-368.

HAUSER O. 1916 - La Micoque. Die Kultur einer neuen Diluvialrasse. Leipzig : Verlag von Veit \& Comp., 58p., $10 \mathrm{pl}$.

HERISSON D., BRENET M., CLIQUET D., MONCEL M.H., RICHTER J., SCOTT B., VAN BAELEN A., DI MODICA K., DE LOECKER D., ASHTON N., BOURGUIGNON L., DELAGNES A., FAIVRE J.-Ph., FOLGADO-LOPEZ M., LOCHT J.-L., POPE M., RAYNAL J.-P., ROEBROEKS W., SANTAGATA C., TURQ A., VAN PEER P. 2016 - The emergence of the Middle Palaeolithic in north-western Europe and its southern fringes. Quaternary International, 411, p. 233-283.

HERNANDEZ M., MERCIER N., BERTRAN P., COLONGE D., LELOUVIER L.-A. 2012 - Premiers éléments de datation des industries du Pléistocène moyen (Acheuléen Paléolithique moyen ancien) de la région pyrénéogaronnaise : une approche géochronologique pluriméthodes (TL, OSL et TT-OSL) des sites de Duclos et Romentères. Paleo, n²3, p. 155-170.

HERNANDEZ M., MERCIER N., RIGAUD J.-Ph., TEXIER J.-P., DELPECH F. 2014 - A revised chronology for the Grotte Vaufrey (Dordogne, France) based on TT-OSL dating of sedimentary quartz. Journal of human evolution, 75 , p. $53-63$.

JARRY M., COLONGE D., LELOUVIER L.-A., MOURRE V. (Dir.) 2007 - Les Bosses (Lamagdelaine, Lot, France) : un gisement paléolithique moyen antérieur à l'avant-dernier interglaciaire sur la moyenne terrasse du Lot. Soc. Préhist. Franç., Travaux 7, 158 p.

LAVILLE H. 1975 - Climatologie et chronologie du Paléolithique en Périgord. Etude sédimentologique de dépôts en grottes et sous abris. Marseille : Editions du laboratoire de Paléontologie humaine et de Préhistoire, Etudes quaternaires, géologie, paléontologie, préhistoire, mémoire ; 4, $422 \mathrm{p}$.

LEPOT M. 1993 - Approche techno-fonctionnelle de l'outillage moustérien. Essai de classification des parties actives en termes d'efficacité technique. Application de la couche M2e sagittale du grand abri de la Ferrassie (fouille 
Henri Delporte). Paris : Université Paris X - Nanterre, Mémoire de Maîtrise, 2 tomes, $170 \mathrm{p}$.

LUMLEY H. de (Dir.) 2004 - Le sol d'occupation acheuléen de l'unité archéostratigraphique UA 25 de la grotte du Lazaret, Edisud, Nice, 491 p.

MATHIAS C. 2016 - After the Lower Palaeolithic : Lithic ramification in the early Middle Palaeolithic of Orgnac 3, layer 2 (Ardèche, France). Quaternary International, 411, p. 193-201

MELLARS P. 1989 - Chronologie du Moustérien du SudOuest de la France : actualisation du débat. L'Anthropologie, t. 43, p. 53-72.

MONCEL M.-H. 1999 - Les assemblages lithiques du site Pléistocène moyen d'Orgnac 3 (Ardèche, Moyenne vallée du Rhône, France). E.R.A.U.L., 89, 446 p.

MONCEL M.-H. (Dir.) 2008 - Le site de Payre. Occupations humaines dans la vallée du Rhône à la fin du Pléistocène moyen et au début du Pléistocène supérieur. Société préhistorique française, mémoire XLVI, $336 \mathrm{p}$.

MONCEL M.-H., MOIGNE A.-M., COMBIER J. 2012 Towards the Middle Palaeolithic in Western Europe: the case of Orgnac 3 (southeastern France). Journal of Human Evolution, $\mathrm{n}^{\circ} 63$, p. 103-124.

MONCEL M.-H., FERNANDES P., CHACON M. G., DE LOMBRERA HERMIDA A., MENENDEZ GRANDA L., YOUCEF S., MOIGNE A.-M., PATOU-MATHIS M., DAUJEARD C., RIVALS F., THEODOROPOULOU A., VALLADAS H., MERCIER N., BAHAIN J.-J., VOINCHET P., FALGUĖRES C., MICHEL V., GUANJUN C., YOKOYAMA Y., COMBIER J. 2014 Emergence et diversification des stratégies au Paléolithique moyen ancien (350 000 à 120000 ans) dans la vallée du Rhône (France). Les sites d'Orgnac 3 et Payre. In : Brenet M., Bourguignon L., Jarry M. (Eds.), Emergence et Diversité Des Techno-Complexes Du Paléolithique Moyen Ancien. Relations Entre Productions de Débitage et de Façonnage, Session $\mathrm{C}$ Du XXVII ${ }^{\mathrm{e}}$ Congrès Préhistorique de France, 31 Mai, 5 Juin 2010. Société Préhistorique Française, Bordeaux, Les Eyzies, p. 59-81.

MOURRE V., COLONGE D. 2007 - Et si l'Acheuléen méridional n'était pas là où on l'attendait ? In : Un siècle de construction du discours scientifique en préhistoire, Avignon 21-258 septembre 2004, Congrès du centenaire de la SPF, 3, p. 68-78.

ORTEGA I., LOCHT J.-L., SORESSI M., RIOS-GARAIZAR J., BOURGUIGNON L., BLASER F., GOVAL E., GRIGOLETTO F., SELLIER N. 2013 - La produccion Laminar durante el Paleolitico medio en el norte y el sudoeste de Francia : el aporte de la experimentacion. In : Palomo A., Piqué R., Terradas X. (Eds.) Experimentacion en arqueologia. Estudio y difusion del pasado, p. 219-228.
PERESANI M. 1998 - La variabilité du débitage discoïde dans la grotte de Fumane (Italie du Nord). Paleo, $\mathrm{n}^{\circ} 10$, p. $123-146$

PEYRONY D. 1938 - La Micoque. Les fouilles récentes. Leur signification. Bulletin de la Société préhistorique française, vol. 35, 6, p. 257-283.

RIGAUD J.-Ph., TEXIER P.-J. 1981 - À propos des particularités techniques et typologiques du gisement des Tares, Commune de Sourzac (Dordogne). Bull. de la Soc. Préhist. Franç., t. 78, fasc. 4, p. 109-117

RIGAUD J.-Ph. (Dir.) 1988 - La grotte Vaufrey : paléoenvironnements, chronologie, activités humaines. Mémoires de la Société préhistorique française, XIX, $616 \mathrm{p}$.

ROLLAND N. 1988 - Variabilité du Paléolithique moyen : nouveaux aspects. In : Farizy C. (Dir.) Paléolithique moyen récent et Paléolithique supérieur ancien en Europe : ruptures et transitions. Examen critique des documents archéologiques, actes du colloque international de Nemours, 9-11 mai 1988, APRAIF, mémoires du musée de Préhistoire d'lle-de-France, $n^{\circ} 3$, p. 69-76.

ROSENDAHL G. 2006 - Les couches supérieures de la Micoque (Dordogne). Paleo, n¹8, p. 161-192.

SÉRONIE-VIVIEN M., SÉRONIE-VIVIEN M.-R. 1987 - Les Silex du Mésozoïque nord-aquitain. Approche géologique de l'étude des silex pour servir à la recherche préhistorique. Supplément au tome XV, Bulletin de la Société Linéenne de Bordeaux, $135 \mathrm{p}$.

TAVOSO A. 1978 - Le Paléolithique inférieur et moyen du Haut-Languedoc. Gisements de terrasses alluviales du Tarn, du Dadou, de l'Agout, du Sor et de Fresquel. Etudes quaternaires, mémoire $n^{\circ} 5$, éditions du laboratoire de paléontologie humaine et de préhistoire, Université de Provence, $403 \mathrm{p}$.

TEXIER J.-P.(Dir.) 2006 - Sédimentogénèse des sites préhistoriques classiques du Périgord. Pôle International de la Préhistoire, Edition numérique, 83 p.

TEXIER J.-P., BERTRAN P. 1993 - Nouvelle interprétation paléo-environnementale et chrono-stratigraphique du site de La Micoque (Dordogne). Implications archéologiques. Comptes Rendus de l'Académie des Sciences de Paris, t. 316, série II, p. 1611-1617.

TURQ A., BRENET M., COLONGE D., JARRY M., LELOUVIER L.-A., O'FARELL M., JAUBERT J. 2010 - The first human occupations in southwestern France : A revised summary twenty years after the Abbeville/Saint-Riquier colloquium. Quaternary International, 223-224, p. 383-398.

TURQ A., FAIVRE J.-Ph., GRAVINA B., BOURGUIGNON L. 2017 - Building models of Neanderthal territories for raw material transport in the Aquitaine Basin (southwestern France), Quaternary International, vol. 433, part. b, p. 88-101. 
VALLADAS H., MERCIER N., FALGUĖRES C., BAHAIN J.-J. 1999 - Contribution des méthodes nucléaires à la chronologie des cultures paléolithiques entre 300000 et 35000 BP. Gallia Préhistoire, vol. 41, p. 153-166.

VIALLET C. 2016 - Potentiel fonctionnel des outils bifaciaux au Pléistocène moyen en contexte méditerranéen. Analyse de la structure et des macro-traces des séries bifaciales de la Caune de l'Arago, Terra Amata, Orgnac 3 et du Lazaret. Perpignan : Université de Perpignan Via-Domitia, Thèse de Doctorat, 398 p. 